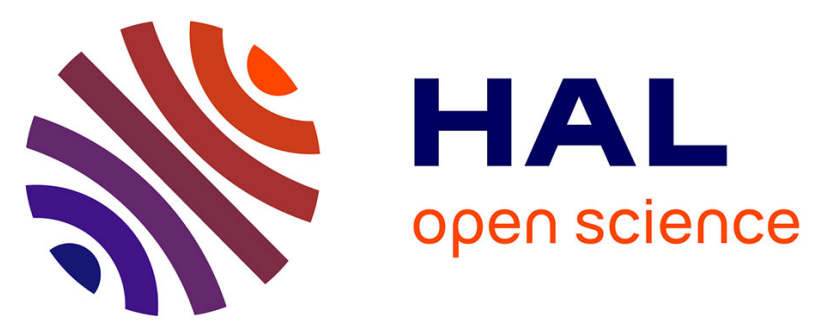

\title{
Monte Carlo implementation of Schiff's approximation for estimating radiative properties of homogeneous, simple-shaped and optically soft particles: Application to photosynthetic micro-organisms
}

Julien Charon, Stéphane Blanco, Jean-François Cornet, Jérémi Dauchet, Mouna El-Hafi, Richard A Fournier, Mira Kaissar Abboud, Sebastian Weitz

\section{To cite this version:}

Julien Charon, Stéphane Blanco, Jean-François Cornet, Jérémi Dauchet, Mouna El-Hafi, et al.. Monte Carlo implementation of Schiff's approximation for estimating radiative properties of homogeneous, simple-shaped and optically soft particles: Application to photosynthetic micro-organisms. Journal of Quantitative Spectroscopy and Radiative Transfer, 2016, 172, p. 3-23. 10.1016/j.jqsrt.2015.10.020 . hal-01599991

\section{HAL Id: hal-01599991 \\ https://hal.science/hal-01599991}

Submitted on 7 Nov 2018

HAL is a multi-disciplinary open access archive for the deposit and dissemination of scientific research documents, whether they are published or not. The documents may come from teaching and research institutions in France or abroad, or from public or private research centers.
L'archive ouverte pluridisciplinaire HAL, est destinée au dépôt et à la diffusion de documents scientifiques de niveau recherche, publiés ou non, émanant des établissements d'enseignement et de recherche français ou étrangers, des laboratoires publics ou privés. 


\title{
Monte Carlo implementation of Schiff's approximation for estimating radiative properties of homogeneous, simple-shaped and optically soft particles: Application to photosynthetic micro-organisms

\author{
Julien Charon ${ }^{\mathrm{a}, \mathrm{b}, \mathrm{c}, \mathrm{e}}$, Stéphane Blanco ${ }^{\mathrm{f}, \mathrm{g}}$, Jean-François Cornet ${ }^{\mathrm{d}, \mathrm{e}}$, \\ Jérémi Dauchet ${ }^{\mathrm{d}, \mathrm{e}, *}$, Mouna El Hafi ${ }^{\mathrm{a}, \mathrm{b}}$, Richard Fournier ${ }^{\mathrm{f}, \mathrm{g}}$, \\ Mira Kaissar Abboud ${ }^{\mathrm{e}, \mathrm{h}}$, Sebastian Weitz ${ }^{\mathrm{d}, \mathrm{e}, \mathrm{g}}$
}

\author{
${ }^{a}$ Université Fédérale de Toulouse Midi-Pyrénées, Mines Albi, UMR CNRS 5302, Centre RAPSODEE, Campus Jarlard, F-81013 Albi CT Cedex \\ 09, France \\ ${ }^{\mathrm{b}}$ CNRS, UMR 5302, RAPSODEE, F-81013 Albi, France \\ ${ }^{\mathrm{C}}$ Université Perpignan Via Domitia, ED 305, 52 av. Paul Alduy, 66860 Perpignan Cedex 9, France \\ d Université Clermont Auvergne, ENSCCF, Institut Pascal, BP 10448, F-63000 Clermont-Ferrand, France \\ e CNRS, UMR 6602, IP, F-63171 Aubière, France \\ ${ }_{\mathrm{f}}^{\mathrm{f}}$ Université Paul Sabatier, UMR 5213 - Laboratoire Plasma et Conversion d'Energie (LAPLACE), B â t. 3R1, 118 Route de Narbonne, F-31062 \\ Toulouse cedex 9, France \\ ${ }^{g}$ CNRS, LAPLACE, F-31062 Toulouse, France \\ ${ }^{\mathrm{h}}$ Université Clermont Auvergne, Université Blaise Pascal, Institut Pascal, BP 10448, F-63000 Clermont-Ferrand, France
}

\begin{abstract}
A B S T R A C T
In the present paper, Schiff's approximation is applied to the study of light scattering by large and optically-soft axisymmetric particles, with special attention to cylindrical and spheroidal photosynthetic micro-organisms. This approximation is similar to the anomalous diffraction approximation but includes a description of phase functions. Resulting formulations for the radiative properties are multidimensional integrals, the numerical resolution of which requires close attention. It is here argued that strong benefits can be expected from a statistical resolution by the Monte Carlo method. But designing such efficient Monte Carlo algorithms requires the development of non-standard algorithmic tricks using careful mathematical analysis of the integral formulations: the codes that we develop (and make available) include an original treatment of the nonlinearity in the differential scattering cross-section (squared modulus of the scattering amplitude) thanks to a double sampling procedure. This approach makes it possible to take advantage of recent methodological advances in the field of Monte Carlo methods, illustrated here by the estimation of sensitivities to parameters. Comparison with reference solutions provided by the T-Matrix method is presented whenever possible. Required geometric calculations are closely similar to those used in standard Monte Carlo codes for geometric optics by the computer-graphics community, i.e. calculation of intersections between rays and surfaces, which opens interesting perspectives for the treatment of particles with complex shapes.
\end{abstract}

Keywords:

Monte Carlo method

Integral formulation

Light scattering

Schiff's approximation

Anomalous diffraction approximation

Photobioreactor

\footnotetext{
Abbreviation: FDTD, Finite Difference Time Domain; MOM, Method of Moment; DDA, Discrete Dipole Approximation

* Corresponding author at: Institut Pascal (UMR-CNRS 6602), Campus Universitaire des Cezeaux, Bat. Polytech, 2 Avenue Blaise Pascal, TSA 60206, CS 60026, 63178 AUBIERE CEDEX - France.

E-mail address: jeremi.dauchet@univ-bpclermont.fr (J. Dauchet).
} 


\section{Introduction}

Retrieving the radiative properties of non-spherical particles between the resonance domain and the geometricoptics domain is a significant difficulty encountered in a wide range of radiative studies. The usual numerical methods used to solve Maxwell's equations rigorously, such as Lorenz-Mie, T-Matrix, FDTD, and Volume Integral methods (MOM, DDA), present some practical limitations concerning their implementation for particles which are both large compared with wavelength, and complex-shaped or strongly elongated [1-3]. This is mainly due to computer memory size limitations for methods based on volume or surface discretization, e.g., DDA, and floating point accuracy for methods based on functional decomposition, e.g., T-Matrix. This identified difficulty provides the motivation for current research efforts: on one hand, the domains of applicability of these numerical methods are continuously being extended (see, e.g., [4,5] for recent developments regarding the DDA method and [6] for the II-T-Matrix approach), leading to significant advances (e.g., recent studies of optical properties of blood platelets [7], red blood cells [8], E. coli bacteria [9] or coccoliths [10]). On the other hand, approximate models are being improved (e.g., in the field of geometric optics [1113]). Among them, Van de Hulst's anomalous diffraction approximation [14] has its place in that it is a compromise between accuracy and computational time. It is widely used in the field of atmospheric science $[15,16]$, astrophysics $[14,17]$, biomedical engineering [18] and oceanography [19], for estimating the single-scattering cross sections of optically soft particles. This asymptotic approximation addresses situations where refractive-index contrasts are low between the particle and its surrounding medium (i.e. for soft particles): its validity conditions are

$x \gg 1$

and

$\left|m_{r}-1\right| \ll 1$

where $x=2 \pi a / \lambda_{e}$ is the particle's size parameter, with $a$ being the characteristic length of the particle, $m_{r}$ its complex relative refractive-index and $\lambda_{e}$ the wavelength of incident radiation within the surrounding medium. But a significant restriction of the anomalous diffraction approximation is that it does not allow the phase function to be calculated for the full range of scattering angles $[0, \pi]$ (or, indeed, the asymmetry parameter). This limitation motivated the present investigation of Schiff's approximation, which was developed in the field of quantum mechanics [20] concurrently with the anomalous diffraction approximation. Both approximations are identical, except for that Schiff's approximation includes a description of the scattering amplitude at large scattering angles, which makes it possible to formulate phase functions.

In Section 2 we show that, despite being a significant simplification of the scattering problem, when particle size and orientation distributions are included, the formulation of radiative properties under anomalous diffraction or Schiff's approximation gives multidimensional integrals that are not straightforward to evaluate efficiently, especially in the case of the differential scattering cross-section. Yet, despite the significant number of studies that use this approximation, only little work has been done on its numerical implementation and, to our knowledge, no code is available for the resolution of Schiff's approximation. ${ }^{1}$ In the present paper, we report a numerical implementation based on the most recent methodological advances in the field of the Monte Carlo method (along the line of [22]), including integral formulation [23,24] (in Section 3), sensitivity analysis [25,26] (in Section 3.3) and variance reduction [27] (in Appendix A). The Monte Carlo method is particularly relevant for evaluating the multidimensional integrals of Schiff's approximation since its convergence is dimension-independent [28,29], which is not the case with usual quadrature methods. As a consequence, the calculation times for evaluating the radiative properties averaged over size and orientation distribution are the same as it is for evaluating the properties of an isolated particle with fixed size and orientation. Moreover, the Monte Carlo method allows us to evaluate simultaneously the three cross-sections (extinction, absorption, scattering), their sensitivities to any parameter and the corresponding numerical errors, all that without a significant increase in the calculation times (compared to the evaluation of just one of these quantities). Nevertheless, resolution of Schiff's approximation for the differential cross-section at large scattering angles remains an open question, due to severe convergence difficulties (encountered with both Monte Carlo and quadrature methods) [30]. This well-known difficulty, called the "negative sign problem", is often encountered when solving wave equations (in the present case Helmholtz's equation) with the Monte Carlo method [31]. For this reason, we developed a further simplification of Schiff's approximation for large-angle scattering, which is included in the present study (see Appendix F and [30]). Validation of this large-angle scattering model in Section 4.1 shows appropriated accuracy for studies that are not exclusively focused on backscattering (in such studies, as for example when modelling natural waters/ocean color background [19], other approaches might be preferred). The Monte Carlo algorithms that are used in Section 4 are detailed in Appendix D and Appendix E, and the corresponding codes are made available in Supplementary Material [32] (these codes have been developed for parallel implementation).

The present work was initiated by the study of visible-light transfer within photosynthetic micro-organism suspensions, for the development of photobioreactors devoted to efficient photobiological carbon dioxide fixation and biofuel production [33-36]. These photosynthesis engineering studies rely on the construction of databases to gather accurate knowledge of the radiative properties of micro-algae: absorption, scattering and extinction cross sections, as well as the cumulative of the differential scattering cross-section (i.e. the angular distribution of the scattered power). Constructing such databases not only requires routine procedures for estimating radiative properties, but also demands an analysis of the sensitivities of these properties to micro-organism species, culture conditions,

${ }^{1}$ To our knowledge, the only available anomalous approximation code is a now obsolete code developed by Flatau in 1992 [21]. This code addresses the evaluation of absorption and extinction cross-sections (more precisely absorption and extinction efficiency factors) but it does not include the angular description of the scattering process: neither differential scattering cross-section nor single-scattering phase function nor scattering amplitude is evaluated. 


\begin{tabular}{|c|c|c|c|}
\hline \multicolumn{2}{|c|}{ Roman letters } & $\begin{array}{l}\mathcal{V}_{p} \\
w \\
W\end{array}$ & $\begin{array}{l}\text { volume of the particle }\left(\mathrm{m}^{3}\right) \\
\text { Monte Carlo weight } \\
\text { differential scattering cross section }\left(\mathrm{m}^{2} / \mathrm{sr}\right)\end{array}$ \\
\hline$a$ & characteristic length of the particle (m) & $x$ & particle's size-parameter \\
\hline $\begin{array}{l}\vec{e}_{o} \\
k_{e}\end{array}$ & $\begin{array}{l}\text { particle's orientation } \\
\text { wave number of the surrounding medium }\end{array}$ & \multicolumn{2}{|c|}{ Greek letters } \\
\hline$l$ & $\left(\mathrm{~m}^{-1}\right)$ length of a straight ray passing & $\lambda_{e}$ & $\begin{array}{l}\text { wavelength within the surrounding } \\
\text { medium }(\mathrm{m})\end{array}$ \\
\hline $\mathcal{P}$ & projected surface of the particle $\left(\mathrm{m}^{2}\right)$ & $\theta_{l}$ & limit angle between small and large scattering \\
\hline $\begin{array}{l}m_{r} \\
n_{r}\end{array}$ & $\begin{array}{l}\text { relative complex refractive index } \\
\text { real part of the relative refractive-index } m_{r}\end{array}$ & $\theta_{o}$ & particle's orientation (rad) \\
\hline$p_{X}$ & $\begin{array}{l}\text { probability density function of the random } \\
\text { variable } X\end{array}$ & $\kappa_{r}$ & imaginary part of the relative refractive-index \\
\hline$Q$ & efficiency factor & $\sigma$ & cross section $\left(\mathrm{m}^{2}\right)$ \\
\hline$R$ & aspect ratio & \multirow{2}{*}{\multicolumn{2}{|c|}{ Subscripts }} \\
\hline$r_{e q}$ & radius of the equivalent sphere volume $(\mathrm{m})$ & & \\
\hline$r_{e q}$ & $\begin{array}{l}\text { melume }(\mathrm{m}) \\
\text { volus or the equivalent sphere }\end{array}$ & $a$ & absorption \\
\hline$s$ & $\begin{array}{l}\text { width parameter of the log-normal } \\
\text { distribution }\end{array}$ & $\begin{array}{l}\text { ext } \\
s\end{array}$ & $\begin{array}{l}\text { extinction } \\
\text { scattering }\end{array}$ \\
\hline$S$ & complex scattering amplitude $\left(\mathrm{m}^{2}\right)$ & & \\
\hline
\end{tabular}

incident light fluxes and spectral distribution, in order to design relevant tabulation of size parameter, shape and complex refractive index (see Section 3.3). The task is challenging since estimating and analyzing the radiative properties of micro-algae either implies highly specialized experiments [34], or the resolution of Maxwell's equations for particles with types of heterogeneities, sizes and shapes for which the usual numerical methods are yet impracticable in most cases [30,2,1,3] (both experimental and numerical approaches complementing each other). Therefore, the use of lightscattering approximations is, to date, necessary and Schiff's approximation has been demonstrated to be one of the best available models for the study of photosynthetic microorganisms [30]: equivalent-sphere size parameters range from $x=10$ to $x=200$, relative refractive indices are typically from $m_{r}=1.01-i 0$ to $m_{r}=1.1-i 5 \cdot 10^{-2}[34,30]$ and Schiff's approximation makes it possible to account for their complex and elongated shapes (up to 50 times longer than wide), including complete angular description of the scattering process (in addition to the absorption and extinction crosssections). In a recent publication [30] we proposed and validated a generic methodological chain for the predictive determination of micro-algae's radiative-properties based on Schiff's approximation for equivalent homogeneous particles with cylindrical and spheroidal shapes whose size distribution, aspect ratio and refractive index are determined by analyzing the morphology and photosynthetic-pigment content of micro-organisms. Our numerical implementation of Schiff's approximation is a key feature of this methodological chain (but was not described in [30]) because it can obtain calculation times and accuracies that are compatible with the databases that we aim to produce and distribute for the purpose of photobioreactor studies (see Section 4). We report these numerical developments separately in the present paper because they are not restricted to the study of photosynthetic micro-organisms: they address any application of Schiff's approximation for homogeneous spheroids and cylinders.

The extension of our approach to the treatment of particles with more complex shapes than cylinder and spheroid is discussed in Section 5 and appears to be conceptually rather straightforward thanks to the computational tools presented in [22]. Finally, this work lays the foundations for subsequent investigations of the convergence difficulties encountered at large scattering angles (or when solving Helmholtz's equation in general) using the zero variance approach $[23,37,38]$.

\section{Schiff's approximation}

The mathematical demonstration of Schiff's approximation is fully detailed in [20] and the corresponding physical pictures were clearly explained by Van de Hulst [14] in the context of the anomalous diffraction approximation. Without re-demonstrating Schiff's approximation, we recall hereafter its fundamental mathematical and physical bases and give the resulting general expressions in the context of lightscattering by soft particles.

In his seminal article [20], Schiff developed an approximation for the study of high-energy electron scattering by heavy nuclei. He addresses the wave function $\psi$ solution of the Schrödinger equation

$\left(\nabla^{2}+k_{e}^{2}-U\right) \psi=0$

with the asymptotic functional form

$\psi \rightarrow \exp \left(i k_{e} \vec{e}_{i} \cdot \vec{r}\right)+\frac{\exp \left(i k_{e} r\right)}{r} f\left(\vec{e}_{s}, \vec{e}_{i}\right)$

where $r$ is the magnitude of the location vector $\vec{r}$, $\hbar^{2} U / 2 m=V$ is the energy of the scattering potential $U$, 
$\hbar^{2} k_{e}^{2} / 2 m$ is the kinetic energy of the incident particle with mass $m$, incident direction $\vec{e}_{i}$ and wave number $k_{e}=2 \pi / \lambda_{e}$, and $f\left(\vec{e}_{s}, \vec{e}_{i}\right)$ is the scattering amplitude in direction $\vec{e}_{s}$. This study was conducted under the following assumptions:

(1) $|V| \ll\left(\mathcal{E}-m c^{2}\right)=\hbar^{2} k_{e}^{2} / 2 m$, where $\mathcal{E}$ is the total energy of the incident particle.

(2) $k_{e} a \gg 1$, where $a$ is the characteristic length corresponding to a significant variation of the potential $U$.

The transposition to our study is straightforward: using the scalar description of electromagnetic wave propagation (which neglects depolarization effects), we address the electric field $E$ solution of the Helmholtz equation $[14,3,39]$

$\left(\nabla^{2}+k_{e}^{2} m_{r}^{2}\right) E=0$

with asymptotic functional form (far field zone)

$E \rightarrow \exp \left(i k_{e} \vec{e}_{i} \cdot \vec{r}\right)+\frac{\exp \left(-i k_{e} r\right)}{-i r} S\left(\vec{e}_{s}, \vec{e}_{i}\right)$

where $k_{e}$ is the wave number of an incident plane wave with propagation direction $\vec{e}_{i}, m_{r}$ is the particle's relative refractive-index and $S\left(\vec{e}_{s}, \vec{e}_{i}\right)$ is the scattering amplitude in direction $\vec{e}_{s}$. Therefore, solutions obtained in [20] (as well as their demonstration) can directly be used in our context, only replacing $f\left(\vec{e}_{s}, \vec{e}_{i}\right)$ with $-i S\left(\vec{e}_{s}, \vec{e}_{i}\right), U$ with $-k_{e}^{2}\left(m_{r}^{2}-1\right)$ and $i$ with $-i$ in order to respect our convention for the complex amplitudes, i.e. for Fourier's transform. In this transposition, Schiff's approximation corresponds to the following assumptions:

(a) assumption (1) corresponds to low refractive-index contrasts

$\left|m_{r}^{2}-1\right| \ll 1$

a

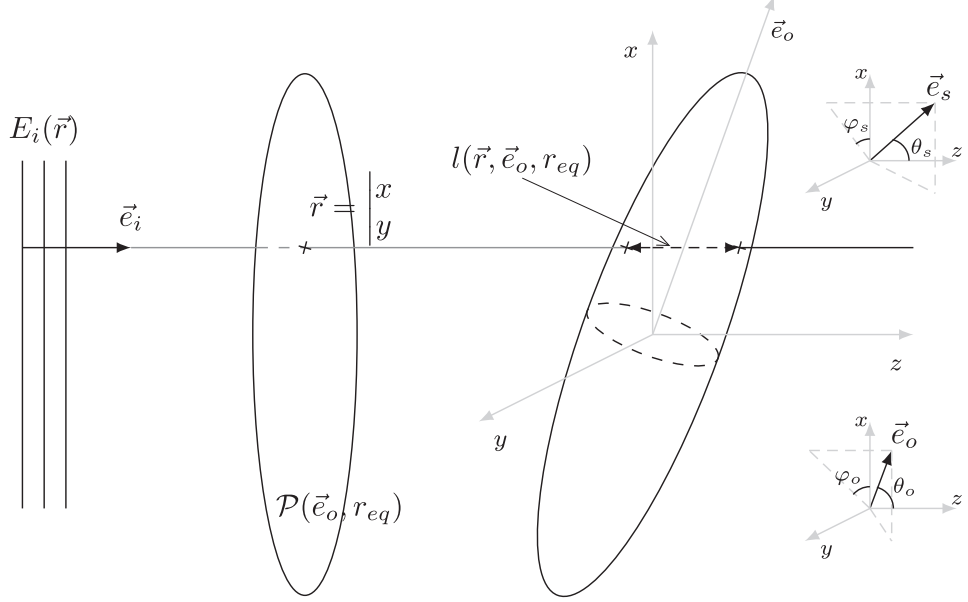

(b) assumption (2) corresponds to large particles compared to the wavelength (i.e. large size parameters $\left.x=k_{e} a\right)$

$x \gg 1$

where $a$ is the characteristic dimension of the particle (its radius for a sphere).

The demonstration proposed by Schiff consists in applying the stationary phase approximation to the infinite Born-series expansion of the scattering amplitude. The Born series is an integral over the infinite dimensional space $\mathcal{V}_{p} \times \mathcal{V}_{p} \times \mathcal{V}_{p} \times \cdots$, where $\mathcal{V}_{p}$ is the volume of the particle [3]. The major difficulty when solving this integral is that the integrand rapidly oscillates within $\mathcal{V}_{p}$ when particles are large compared to incident wavelength. Schiff takes advantage of situations where particles are large and refractive-index contrasts are low (i.e. when the validity conditions (a) and (b) are satisfied) by explicitly exhibiting regions of the integration domain that significantly contribute to the scattering amplitude, and other regions where the integrand is rapidly oscillating around zero, leading to second-order contributions that are neglected. Only the stationary phase regions, where the integrand is not oscillating, are retained in the expression of the scattering amplitude under Schiff's approximation. Finally, in the context of light scattering, the first-order Taylor series approximation $k_{e}^{2}\left(m_{r}^{2}-1\right) \simeq 2 k_{e}^{2}\left(m_{r}-1\right)$ around $m_{r}=1$ has to be included in Schiff's results so that the order of approximation for the scattering potential is consistent with that for the scattering amplitude $S$ (see [39]). This demonstration leads to the validity conditions (Eqs. (1) and (2)), and to the definition of an angle $\theta_{l}$ that sets the limit between small and large scattering angles [30,20]:

$\theta_{l}=\sqrt{\frac{2}{\tilde{x}}}$

where $\tilde{x}=2 \pi \tilde{a} / \lambda_{e}$ is the size parameter calculated for the b

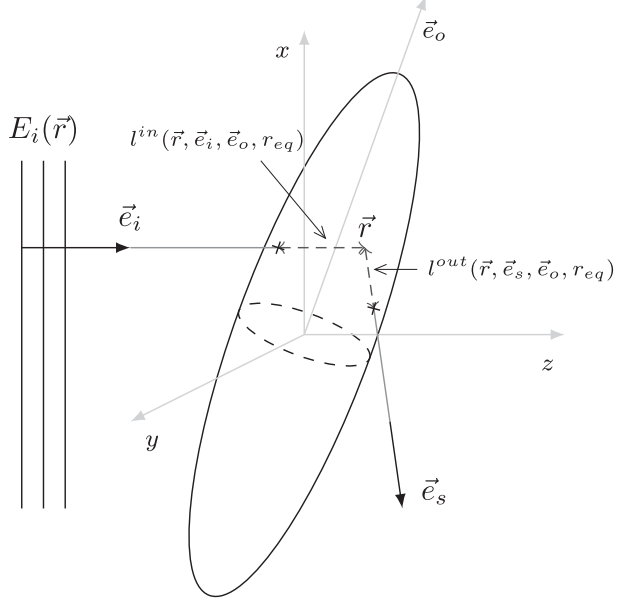

Fig. 1. Schematic view defining the geometric quantities that appear in Schiff's approximation. A rotationally symmetric particle is submitted to an incident plane wave $E_{i}$ with propagation direction $\vec{e}_{i}$. The orientation of the particle is defined by the direction of its rotational axis $\vec{e}_{o}$ (corresponding to the two spherical angles $\theta_{o}$ and $\varphi_{o}$ ). $\vec{e}_{s}$ is the considered scattering direction (corresponding to $\theta_{s}$ and $\varphi_{s}$ ). (a) For small scattering angles $\theta_{s}$, we consider straight rays starting at location $\vec{r}(x, y)$ of the projected surface $\mathcal{P}\left(\vec{e}_{o}, r_{e q}\right)$ of the particle seen from the incident direction (where $r_{e q}$ is the radius of the volume-equivalent sphere). $l\left(\vec{r}, \vec{e}_{o}, r_{e q}\right)$ is the crossing length of such a ray passing through the particle until it exits the particle. (b) For large scattering angles $\theta_{s}$, we consider rays entering the particle with direction $\vec{e}_{i}$, scattered at a location $\vec{r}(x, y)$ inside the particle volume, and leaving it with direction $\vec{e}_{s} . l^{i n}\left(\vec{r}, \vec{e}_{i}, \vec{e}_{o}, r_{e q}\right)$ is the length of the ray from its entry into the particle to $\vec{r}$ and $l^{\text {out }}\left(\vec{r}, \vec{e}_{i}, \vec{e}_{o}, r_{e q}\right)$ the length from $\vec{r}$ to its exit of the particle. 
smallest characteristic dimension $\tilde{a}$ of the scatterer (this dimension controls the angular aperture of the scattered power).

For small scattering angles $\left(\theta_{s} \lesssim \theta_{l}\right)$, the integration domain of Born's series reduces to the projected surface $\mathcal{P}$ of the particle and the resulting expressions are identical to that given by the anomalous diffraction approximation. For a homogeneous axisymmetric particle with fixed size $r_{e q}$ and orientation $\vec{e}_{o}$ located within a non-absorbing surrounding medium with homogeneous refractive index $n_{e}$, formulation of the scattering amplitude $[20,14]$ is given by Eq. (8) where we choose to characterize the particle's size and orientation respectively by the radius $r_{e q}$ of its volume equivalent sphere and the unit vector $\vec{e}_{o}$ along its symmetry axis (see Fig. 1).

$$
\begin{aligned}
& S\left(\vec{e}_{s}, \vec{e}_{i}, \vec{e}_{o}, r_{e q}\right)=\frac{k_{e}}{2 \pi} \int_{\mathcal{P}\left(\vec{e}_{o}, r_{e q}\right)} \exp \left[i k _ { e } \theta _ { s } \left(x \cos \varphi_{s}\right.\right. \\
& \left.\left.\quad+y \sin \varphi_{s}\right)\right]\left(1-\exp \left[-i k_{e}\left(m_{r}-1\right) l\left(\vec{r}, \vec{e}_{o}, r_{e q}\right)\right]\right) d \vec{r}
\end{aligned}
$$

where $k_{e}=2 \pi / \lambda_{e}$ is the wave number of incident radiation with wavelength $\lambda_{e}, \vec{r}=(x, y)$ is a location on the projected surface $\mathcal{P}\left(\vec{e}_{o}, r_{e q}\right)$ of the particle seen from the incident direction $\vec{e}_{i}, l\left(\vec{r}, \vec{e}_{o}, r_{e q}\right)$ is the crossing length through the particle for the straight ray along $\vec{e}_{i}$ that intersects $\mathcal{P}\left(\vec{e}_{o}, r_{e q}\right)$ at location $\vec{r}$, and the scattering direction $\vec{e}_{s}$ is defined by the scattering angles $\theta_{s}$ and $\varphi_{s}$ (see Fig. 1). Using the optical theorem $\hat{\sigma}_{\text {ext }}=\left(4 \pi / k_{e}\right)$ $\mathfrak{R S}\left(\vec{e}_{i}, \vec{e}_{i}, \vec{e}_{o}, r_{e q}\right)$ and the definition $\hat{W}_{s}=|S|^{2}$ for the differential scattering cross-section [14,39], the formulation of the radiative properties is (as for the anomalous diffraction approximation)

$$
\begin{aligned}
& \hat{\sigma}_{e x t}\left(\vec{e}_{o}, r_{e q}\right)=2 \int_{\mathcal{P}\left(\vec{e}_{o}, r_{e q}\right)} 1-e^{-k_{e} k_{r} l\left(\vec{r}, \vec{e}_{o}, r_{e q}\right)} \\
& \quad \times \cos \left(k_{e}\left(n_{r}-1\right) l\left(\vec{r}, \vec{e}_{o}, r_{e q}\right)\right) d \vec{r} \\
& \hat{\sigma}_{a}\left(\vec{e}_{o}, r_{e q}\right)=\int_{\mathcal{P}\left(\vec{e}_{o}, r_{e q}\right)} 1-e^{-2 k_{e} k_{r} l\left(\vec{r}, \vec{e}_{o}, r_{e q}\right)} d \vec{r} \\
& \hat{\sigma}_{s}\left(\vec{e}_{o}, r_{e q}\right)=\hat{\sigma}_{e x t}-\hat{\sigma}_{a} \\
& \hat{W}_{s}\left(\vec{e}_{s}, \vec{e}_{i}, \vec{e}_{o}, r_{e q}\right) \\
& =\left(\frac{k_{e}}{2 \pi}\right)^{2} \mid \int_{\mathcal{P}}\left(\vec{e}_{o}, r_{e q}\right) \exp \left[i k_{e} \theta_{s}\left(x \cos \varphi_{s}+y \sin \varphi_{s}\right)\right] \\
& \quad \times\left.\left(1-\exp \left[-i k_{e}\left(m_{r}-1\right) l\left(\vec{r}, \vec{e}_{o}, r_{e q}\right)\right]\right) d \vec{r}\right|^{2}
\end{aligned}
$$

where $\hat{\sigma}_{\text {ext }}, \hat{\sigma}_{a}$ and $\hat{\sigma}_{s}$ are respectively the extinction, absorption and scattering cross-sections, $\hat{W}_{s}$ is the differential scattering cross-section and $n_{r}$ and $\kappa_{r}$ are respectively the real and imaginary part of the particle's relative refractive index ${ }^{2}: m_{r}=n_{r}-i \kappa_{r}$. As further detailed in [14], these formulations of the radiative properties can be

\footnotetext{
${ }^{2}$ Let us note $n$ the real part of particle's refractive index and $\kappa$ its imaginary part. Then, $n_{r}=n / n_{e}$ and $\kappa_{r}=\kappa / n_{e}$, where $n_{e}$ is the refractive index of the surrounding medium. The surrounding medium does not absorb radiation, therefore the imaginary part of its refractive index is zero.
}

interpreted in the light of simple physical pictures that are directly related to the validity conditions (Eqs. (1) and (2)):

- $x \gg 1$, which ensures that the ray picture of the geometrical optics is applicable,

- $\left|m_{r}-1\right| \ll 1$, which ensures that the reflection and refraction of incident light by the particle can be ignored: the incident rays cross the particle in a straight line, they do not bend when entering and leaving the particle.

Therefore, a straight ray that crosses the particle with crossing length $l$ is attenuated and phase-shifted according to $\exp \left(-i k_{e}\left(m_{r}-1\right) l\right)=\exp \left(-k_{e} \kappa_{r} l\right) \exp \left(-i k_{e}\left(n_{r}-1\right) l\right)$ compared to an incident ray that does not pass through the particle. These attenuations and phase shifts give the electric field in a plane perpendicular to $\vec{e}_{i}$ at coordinate $z$ just beyond the particle. Then, Schiff's approximation consists in applying Huygens' principle to this plane in order to propagate the electromagnetic wave and account for interferences in the far field region [14] (which leads to the Fraunhofer-like diffraction pattern $\int_{\mathcal{P}} \exp \left[i k_{e} \theta_{s}\right.$ $\left.\left(x \cos \varphi_{s}+y \sin \varphi_{s}\right)\right] \cdots$ in Eq. (12)).

For large scattering angles $\left(\theta_{s} \gtrsim \theta_{l}\right)$, formulation of the scattering amplitude under Schiff's approximation is the following integral over the volume $\mathcal{V}_{p}$ of the particle [20]:

$$
\begin{aligned}
S\left(\vec{e}_{s}, \vec{e}_{i}, \vec{e}_{o}, r_{e q}\right)= & \frac{i k_{e}^{2}}{2 \pi}\left(m_{r}-1\right) \times \int_{\mathcal{V}_{p}\left(\vec{e}_{o}, r_{e q}\right)} \exp (i \vec{q} \cdot \vec{r}) \\
& \exp \left[-i k_{e}\left(m_{r}-1\right)\left(l^{i n}\left(\vec{r}, \vec{e}_{i}, \vec{e}_{o}, r_{e q}\right)\right.\right. \\
& \left.\left.+l^{\text {out }}\left(\vec{r}, \vec{e}_{s}, \vec{e}_{o}, r_{e q}\right)\right)\right] d \vec{r}
\end{aligned}
$$

where $\vec{q}=k_{e}\left(\vec{e}_{s}-\vec{e}_{i}\right), l^{i n}\left(\vec{r}, \vec{e}_{i}, \vec{e}_{o}, r_{e q}\right)$ is the length of an incident ray between its entry into the particle and the location $\vec{r}$ within the particle, and $l^{\text {out }}\left(\vec{r}, \vec{e}_{s}, \vec{e}_{o}, r_{e q}\right)$ is the length of a ray starting from the location $\vec{r}$ with the scattering direction $\vec{e}_{s}$ until it exits the particle (see Fig. 1b). Other notations have been defined after Eq. (12). When studying light-scattering within the scalar description of wave propagation, pure geometrical effects due to the vectorial nature of the electromagnetic fields must be accounted for. Indeed, the scalar description only assumes that parallel and perpendicular components of the field are solutions of the same equation, but projection of these components on the plane perpendicular to $\vec{e}_{s}$ must be treated. In the present context (i.e. radiative properties calculation for incoherent non-polarized incident fields) these effects come down to the multiplication of $\hat{W}_{s}$ by the pre-factor $\left(\cos ^{2}\left(\theta_{s}\right)+1\right) / 2$ (as for Rayleigh-Gans approximation [39]), which leads to:

$$
\begin{aligned}
& \hat{W}_{s}\left(\vec{e}_{s}, \vec{e}_{i}, \vec{e}_{o}, r_{e q}\right)=\left(\frac{k_{e}^{2}}{2 \pi}\right)^{2}\left|m_{r}-1\right|^{2} \frac{1+\cos ^{2}\left(\theta_{s}\right)}{2} \\
& \times \mid \begin{array}{l}
\int_{\mathcal{V}_{p}}\left(\vec{e}_{o}, r_{e q}\right) \\
\quad \times \exp (i \vec{q} \cdot \vec{r})
\end{array} \\
& \times \exp \left[-\left.i k_{e}\left(m_{r}-1\right)\left(l^{\text {in }}\left(\vec{r}, \vec{e}_{i}, \vec{e}_{o}, r_{e q}\right)+l^{\text {out }}\left(\vec{r}, \vec{e}_{s}, \vec{e}_{o}, r_{e q}\right)\right] d \vec{r}\right|^{2}\right.
\end{aligned}
$$

The physical pictures associated with large-angle scattering remain closely similar to those for small angles, but the deviation of optical paths by the particle can no longer be neglected. This deviation is not due to refraction and 
reflection at the interface between the particle and the surrounding medium (we stay within the same pictures as before) but it is due to the interaction between the incident radiation and the material constituting the particle: attenuation and phase shift are now calculated according to the crossing length $l^{\text {in }}+l^{\text {out }}$ of "single-scattering" paths (see Fig. 1b). The above equation is a significant contribution of Schiff's approximation for the evaluation of soft particles' radiative properties since, in conjunction with Eq. (12), it gives an expression of the single-scattering phase function (which is not the case with the anomalous diffraction approximation). Nevertheless, numerically estimating Eq. (14) requires tackling severe convergence difficulties $^{3}$ (negative sign problem), which is a research field in itself that is out of the scope of the present paper [31]. But the physical pictures of Schiff's approximation at large angles remain available and, although numerically tedious to evaluate, Eq. (14) has interesting mathematical properties. Based on these pictures and mathematical properties, a simplified but fully practical description of large-angle scattering is derived in Appendix $\mathrm{F}$ for the study of photosynthetic micro-organisms cultivated in photobioreactors. In the following, we use this simplified model (which is reported hereafter in Eq. (18)) and the resulting phase functions are validated against T-Matrix results in Section 4.

Up to this point, we have considered a particle with fixed size and orientation. Let us now average the previous results over the particle size distribution $p_{R_{e q}}\left(r_{e q}\right)$ and orientation distribution $p_{\vec{F}}\left(\vec{e}_{o}\right)$ in order to obtain the radiative properties of a suspension of particles under the assumption of independent scattering:

$$
\begin{aligned}
& \sigma_{e x t}=\int_{4 \pi} d \vec{e}_{o} p_{\vec{E}_{o}}\left(\vec{e}_{o}\right) \int_{0}^{\infty} d r_{e q} p_{R_{e q}}\left(r_{e q}\right) \hat{\sigma}_{e x t}\left(\vec{e}_{o}, r_{e q}\right) \\
& \sigma_{a}=\int_{4 \pi} d \vec{e}_{o} p_{\vec{E}_{o}}\left(\vec{e}_{o}\right) \int_{0}^{\infty} d r_{e q} p_{R_{e q}}\left(r_{e q}\right) \hat{\sigma}_{a}\left(\vec{e}_{o}, r_{e q}\right) \\
& \sigma_{s}=\sigma_{e x t}-\sigma_{a}
\end{aligned}
$$

$W_{s}\left(\theta_{s}\right)=\int_{4 \pi} d \vec{e}_{o} p_{\vec{E}_{o}}\left(\vec{e}_{o}\right) \int_{0}^{\infty} d r_{e q} p_{R_{e q}}\left(r_{e q}\right) \hat{W}_{s}\left(\vec{e}_{s}, \vec{e}_{i}\right)$

for $\left(\theta_{s} \leq \theta_{l}\right)$

$W_{s}\left(\theta_{s}\right)=\frac{A}{\sin ^{B}\left(\theta_{s} / 2\right)} \frac{1+\cos ^{2}\left(\theta_{s}\right)}{2}$ for $\theta_{s} \geq \theta_{l}$

where $4 \pi$ is the total solid angle (integration space for orientations) and expressions of $\hat{\sigma}_{\text {ext }}, \hat{\sigma}_{a}$ and $\hat{W}_{s}$ are respectively given in Eqs. (9), (10) and (12). We note that once averaged over the particles' orientation distribution, the differential scattering cross-section (Eq. (17)) for a collection of axisymmetric particles is a function only of the scattering angle $\theta_{s}$ : it does not depend on $\varphi_{s}$ (see Fig. 1

\footnotetext{
${ }^{3}$ We note that similar, but less severe, convergence difficulties are encountered when implementing Eq. (12) (i.e. the anomalous diffraction approximation) for large scattering angles. Although applying Eq. (12) for $\theta>\theta_{l}$ is not physically consistent, we tested it for spheres and recorded results that are by far less accurate than with our simplified model (Eq. (19)), when compared with reference Mie solutions.
}

and [3]). In Eq. (18), $A$ and $B$ are two free parameters that have to be adjusted in order to fulfil the continuity of the differential scattering cross-section at angle $\theta_{l}$ and the equality between the scattering cross-section and the cumulative $C W_{s}$ of the differential scattering cross-section taken at $\pi: \sigma_{s}=C W_{S}(\pi)=2 \pi \int_{0}^{\pi} d \theta_{s} \sin \left(\theta_{S}\right) W_{s}\left(\theta_{s}\right)$ (integration over the total solid angle). From a practical point of view, first $\sigma_{s}, W_{s}\left(\theta_{l}\right)$ and $C W_{s}\left(\theta_{l}\right)$ are evaluated according to Eqs. (17) and (18), then $A$ and $B$ are adjusted as described in Appendix G.

The next paragraphs are devoted to the resolution of Eqs. (15)-(18) with the Monte Carlo method.

\section{Integral formulation of the Monte Carlo algorithms}

In this section, we follow the methodology presented in [22] to develop a statistical formulation of the integral expressions obtained with Schiff's approximation for the radiative properties, in order to solve them using the Monte Carlo method. The resulting algorithms are the direct translation of the physical pictures discussed in Section 2: first, size and orientation of the scatterer are randomly sampled according to its size and orientation distributions, then a straight incident ray is sampled, and finally the attenuation and phase shift due to the ray's path through the particle is computed.

\subsection{Cross sections}

To obtain a statistical description of the cross-sections, we introduce an arbitrary probability density function $p_{\vec{R}}(\vec{r})$ for the location $\vec{r}$ on the projected surface $\mathcal{P}\left(\vec{e}_{o}, r_{e q}\right)$. This can be done by multiplying and dividing the integrands in Eqs. (15)-(17) by $p_{\vec{R}}(\vec{r})$. Applying this methodology to Eq. (19) gives

$$
\begin{aligned}
& \sigma_{e x t}=\int_{4 \pi} d \vec{e}_{o} p_{E_{o}}\left(\vec{e}_{o}\right) \int_{0}^{\infty} d r_{e q} p_{R_{e q}}\left(r_{e q}\right) \\
& \times \int_{\mathcal{P}\left(\vec{e}_{o}, r_{e q}\right)} d \vec{r} p_{\vec{R}}(\vec{r}) \\
& \frac{2\left[1-e^{-k_{e} \kappa_{r} l\left(\vec{r}, \vec{e}_{o}, r_{e q}\right)} \cos \left(k_{e}\left(n_{r}-1\right) l\left(\vec{r}, \vec{e}_{o}, r_{e q}\right)\right)\right]}{p_{\vec{R}}(\vec{r})}
\end{aligned}
$$

which can be written as the Monte Carlo integral formulation

$$
\begin{aligned}
& \sigma_{e x t}=\int_{4 \pi} d \vec{e}_{o} p_{\vec{E}_{o}}\left(\vec{e}_{o}\right) \\
& \int_{0}^{\infty} d r_{e q} p_{R_{e q}}\left(r_{e q}\right) \int_{\mathcal{P}\left(\vec{e}_{o}, r_{e q}\right)} d \vec{r} p_{\vec{R}}(\vec{r}) w_{e x t}\left(\vec{e}_{o}, r_{e q}, \vec{r}\right)
\end{aligned}
$$

where

$w_{e x t}\left(\vec{e}_{o}, r_{e q}, \vec{r}\right)$ 


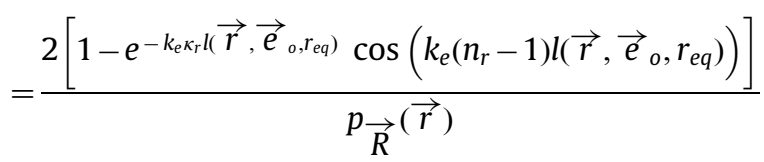

is the Monte Carlo weight function, $p_{\vec{E}_{o}}\left(\vec{e}_{o}\right)$ is the particle's orientation distribution, $p_{R_{e q}}\left(r_{e q}\right)$ is its size distribution and $p_{\vec{R}}(\vec{r})$ is an arbitrary (strictly positive) probability density function. The choice of $p_{\vec{R}}(\vec{r})$ only affects the convergence speed, but the Monte Carlo algorithm will always converge to the value of $\sigma_{\text {ext }}$. In the present work, $p_{\vec{R}}(\vec{r})$ is arbitrarily chosen as a uniform probability density function (the simplest choice):

$p_{\vec{R}}(\vec{r})=\frac{1}{P\left(\vec{e}_{o}, r_{e q}\right)}$

where $P\left(\vec{e}_{o}, r_{e q}\right)$ is the area of the projected surface $\mathcal{P}\left(\vec{e}_{o}, r_{e q}\right)$, i.e. that each point of $\mathcal{P}\left(\vec{e}_{o}, r_{e q}\right)$ has the same probability of being sampled. Injecting Eq. (23) into the general expression of the Monte Carlo weight (Eq. (22)) leads to the specific expression (Eq. (25)) for $w_{\text {ext }}$.

By applying the same methodology to Eqs. (16) and (17), and also keeping the same choice for $p_{\vec{R}}(\vec{r})$, the following Monte Carlo integral-formulation can Đe derived for the three cross-sections $\sigma_{i}$, where subscript $i$ refers either to extinction (ext), absorption (a) or scattering $(s)$ :

$$
\begin{aligned}
\sigma_{i}= & \int_{4 \pi} d \vec{e}_{o} p_{\vec{E}_{o}}\left(\vec{e}_{o}\right) \int_{0}^{\infty} d r_{e q} p_{R_{e q}}\left(r_{e q}\right) \\
& \times \int_{\mathcal{P}\left(\vec{e}_{o}, r_{e q}\right)} d r p_{\vec{R}^{(}}(\vec{r}) w_{i}\left(\vec{e}_{o}, r_{e q}, \vec{r}\right)
\end{aligned}
$$

with $w_{i}$ the Monte Carlo weight functions:

$$
\begin{aligned}
w_{e x t}\left(\vec{e}_{o}, r_{e q}, \vec{r}\right)= & 2 P\left(\vec{e}_{o}, r_{e q}\right) \\
& \times\left[1-e^{-k_{e} \kappa_{r} l\left(\vec{r}, \vec{e}_{o}, r_{e q}\right)}\right. \\
& \left.\times \cos \left(k_{e}\left(n_{r}-1\right) l\left(\vec{r}, \vec{e}_{o}, r_{e q}\right)\right)\right] \\
w_{a}\left(\vec{e}_{o}, r_{e q}, \vec{r}\right)= & P\left(\vec{e}_{o}, r_{e q}\right)\left[1-e^{-2 k_{e} \kappa_{r} l\left(\vec{r}, \vec{e}_{o}, r_{e q}\right)}\right] \\
w_{s}\left(\vec{e}_{o}, r_{e q}, \vec{r}\right)= & w_{e x t}-w_{a}
\end{aligned}
$$

where the location $\vec{r}$ of the projected surface $\mathcal{P}$ only appears within the crossing length $l\left(\vec{r}, \vec{e}_{o}, r_{e q}\right)$ that is known analytically for spheroidal and cylindrical particles (see Fig. 1a for the definition of $l$ and [40], Appendices D and $\mathrm{E}$ for its analytical expression).

The sampling procedure of the corresponding Monte Carlo algorithm is the direct translation of Eq. (24) (see Fig. 2):

- Step 1: An orientation $\vec{e}_{o}$ of the particle is sampled over the total solid angle according to the orientation distribution $p_{\vec{E}_{o}}\left(\vec{e}_{o}\right)$ (any distribution can be used).

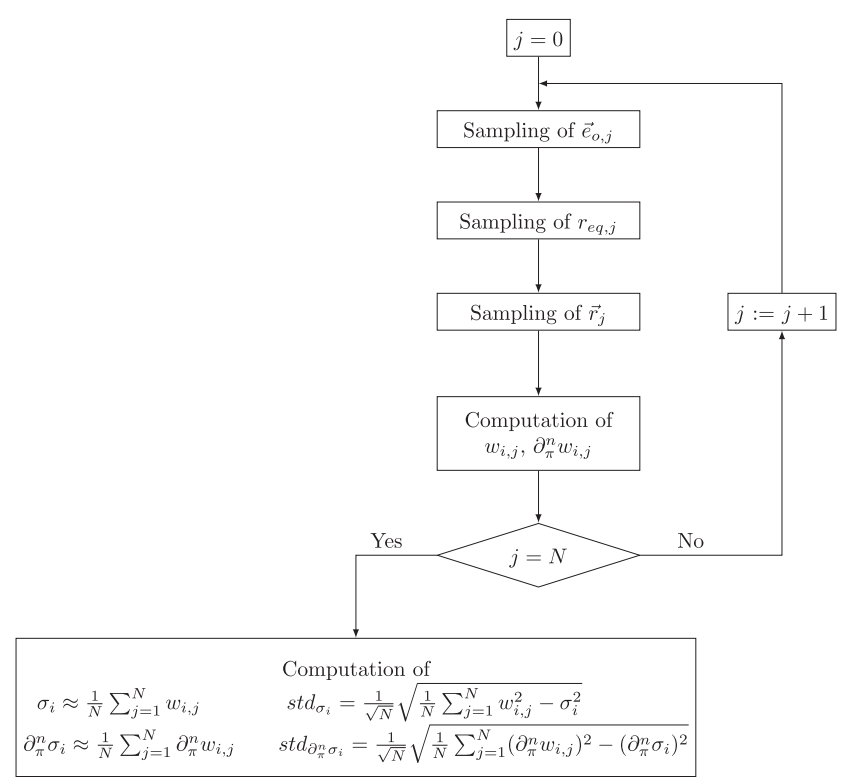

Fig. 2. Monte Carlo algorithm for the simultaneous evaluation of the three cross sections $\sigma_{i}$, their sensitivities $\partial_{\pi}^{n} \sigma_{i}$ to any parameter $\pi$ and the associated standard deviations. The Monte Carlo realization $j$ consists in the following steps: first, an orientation $\vec{e}_{o, j}$ of the particle and a radius $r_{e q, j}$ of the equivalent-volume sphere are sampled. Then, a location $\vec{r}_{j}$ on the projected surface $\mathcal{P}\left(\vec{e}_{o}, r_{e q}\right)$ is sampled. Finally, the Monte Carlo weights $w_{i, j}$ and $\partial_{\pi}^{n} w_{i, j}$ are computed according to the formula given in Section 3.1 and Appendix C. This algorithm is repeated $N$ times and $\sigma_{i}$ and $\partial_{\pi}^{n} \sigma_{i}$ are estimated as the means of the corresponding weights.

- Step 2: A radius $r_{e q}$ for the volume equivalent sphere is sampled over ] $0,+\infty$ [ according to $p_{R_{e q}}\left(r_{e q}\right)$, defining the size of the particle (any distribution can be used).

- Step 3: A location $\vec{r}$ is uniformly sampled over the projected surface $\mathcal{P}\left(\vec{e}_{o}, r_{e q}\right)$ defined by the particle's orientation $\vec{e}_{o}$ and size $r_{e q}$ (which were sampled at Steps 1 and 2).

- Step 4: The crossing length $l\left(\vec{r}, \vec{e}_{o}, r_{e q}\right)$ of the straight ray starting at $\vec{r}$ is computed (see Fig. 1 a).

- Step 5: The Monte Carlo weights $w_{\text {ext }}, w_{a}$ and $w_{s}$ are computed according to Eqs. (25)-(27).

This sampling procedure is repeated $N$ times to get $N$ values of the weights $w_{e x t}, w_{a}$ and $w_{s}$, indexed $w_{e x t, j}, w_{a, j}$ and $w_{s, j}$ respectively. Then, the cross sections are evaluated as $\sigma_{i} \simeq(1 / N) \sum_{j=1}^{N} w_{i, j}$, where $i$ either stands for ext, $a$ or $s$, and the corresponding standard deviations are evaluated as (see [22]):

$\operatorname{std}_{\sigma_{i}}=\frac{1}{\sqrt{N}} \sqrt{\frac{1}{N} \sum_{j=1}^{N} w_{i, j}^{2}-\sigma_{i}^{2}}$.

We note that in this algorithm, the scattering crosssection is not evaluated as the difference $\sigma_{s}=\sigma_{\text {ext }}-\sigma_{a}$ between the extinction and absorption cross-sections, like in Eq. (16), but instead $\sigma_{s}$ is evaluated as the average of the Monte Carlo weights $w_{s, j}=w_{\text {ext }, j}-w_{a, j}$ (see Eq. (26)). Both strategies lead to strictly identical numerical results (because the expectation is a linear operator), but we retained the second strategy because it permits the 
straightforward calculation of the corresponding numerical error using Eq. (28). ${ }^{4}$ Of course this approach is only pertinent because with Monte Carlo method, calculation times for evaluating simultaneously the three crosssections (as in our algorithm) are very similar to those for evaluating just one cross-section.

\subsection{Differential scattering cross-section at small angles}

Hereafter we focus on the integral equation of the differential scattering cross-section $W_{s}$ at small angles (injecting Eq. (12) into Eq. (17)):

$$
\begin{aligned}
W_{s}\left(\theta_{s}\right)= & \int_{4 \pi} d \vec{e}_{o} p_{\vec{E}_{o}}\left(\vec{e}_{o}\right) \int_{0}^{\infty} d r_{e q} p_{R_{e q}}\left(r_{e q}\right)\left(\frac{k_{e}}{2 \pi}\right)^{2} \\
& \times\left|\int_{\mathcal{P}\left(\vec{e}_{o}, r_{e q}\right)} g\left(\vec{r}, \theta_{s}, \varphi_{s}\right) d \vec{r}\right|^{2}
\end{aligned}
$$

where we have introduced

$$
\begin{aligned}
g\left(\vec{r}, \theta_{s}, \varphi_{s}\right)= & \exp \left[i k_{e} \theta_{s}\left(x \cos \varphi_{s}+y \sin \varphi_{s}\right)\right] \\
& \left(1-\exp \left(-i k_{e}\left(m_{r}-1\right) l\left(\vec{r}, \vec{e}_{o}, r_{e q}\right)\right)\right)
\end{aligned}
$$

with the notations of Fig. 1a: we recall that $x$ and $y$ are the coordinates of the location $\vec{r}$ of the projected surface $\mathcal{P}\left(\vec{e}_{o}, r_{e q}\right) ; \theta_{s}$ and $\varphi_{s}$ are the scattering angles.

Our aim here is to design a Monte Carlo algorithm for the direct evaluation of $W_{s}$, without intermediate calculation steps dedicated to the evaluation of the real and imaginary parts of $\int_{\mathcal{p}} g(\vec{r}) d \vec{r}$. Obtaining such a statistical description compatible with the Monte Carlo method implies getting rid of the squared modulus in Eq. (29). This can be done by using the following mathematical trick:

$$
\begin{aligned}
\left|\int_{\mathcal{P}} g(\vec{r}) d \vec{r}\right|^{2} & =\mathfrak{R}^{2} \int_{\mathcal{P}} g(\vec{r}) d \vec{r}+\mathfrak{J}^{2} \int_{\mathcal{P}} g(\vec{r}) d \vec{r} \\
& =\mathfrak{R} \int_{\mathcal{P}} g\left(\vec{r}_{1}\right) d \vec{r}_{1} \times \mathfrak{R} \int_{\mathcal{P}} g\left(\vec{r}_{2}\right) d \vec{r}_{2} \\
+ & \mathfrak{I} \int_{\mathcal{P}} g\left(\vec{r}_{1}\right) d \vec{r}_{1} \times \mathfrak{I} \int_{\mathcal{P}} g\left(\vec{r}_{2}\right) d \vec{r}_{2} \\
& =\int_{\mathcal{P}} d \vec{r}_{1} \int_{\mathcal{P}} d \vec{r}_{2}\left[\mathfrak{R}\left(g\left(\vec{r}_{1}\right)\right) \mathfrak{R}\left(g\left(\vec{r}_{2}\right)\right)\right. \\
& \left.+\mathfrak{I}\left(g\left(\vec{r}_{1}\right)\right) \mathfrak{I}\left(g\left(\vec{r}_{2}\right)\right)\right]
\end{aligned}
$$

where we have omitted the dependency of function $g$ to $\theta_{s}$ and $\varphi_{s}$ for the sake of legibility, and where $\mathfrak{R}$ and $\mathfrak{I}$ are the real and imaginary parts of a complex number. Injecting Eq. (31) into Eq. (29) and introducing arbitrary probability density functions $p_{\vec{R}_{1}}\left(\vec{r}_{1}\right)$ and $p_{\vec{R}_{2}}\left(\vec{r}_{2}\right)$ for the locations $\vec{r}_{1}$ and $\vec{r}_{2}$, as detailed in Section 3.1, we obtain the following Monte Carlo integral formulation:

$W_{s}\left(\theta_{s}\right)=\int_{4 \pi} d \vec{e}_{o} p_{\vec{E}_{o}}\left(\vec{e}_{o}\right) \int_{0}^{\infty} d r_{e q} p_{R_{e q}}\left(r_{e q}\right)$

\footnotetext{
${ }^{4}$ When using the first strategy $\left(\sigma_{s}=\sigma_{e x t}-\sigma_{a}\right)$, the calculation of the corresponding numerical error is not straightforward because the estimator of $\sigma_{s}$ is the difference between two correlated random variables (since the same Monte Carlo realizations are used to compute both $\sigma_{\text {ext }}$ and $\sigma_{a}$ ) and therefore the usual formula $s t d_{\sigma_{\mathrm{s}}} \approx s t d_{\sigma_{\text {ext }}}+s t d_{\sigma_{a}}$ cannot be used.
}

$$
\begin{aligned}
& \times \int_{\mathcal{P}\left(\vec{e}_{o}, r_{e q}\right)} d \vec{r}_{1} p_{R_{1}}\left(\vec{r}_{1}\right) \int_{\mathcal{P}\left(\vec{e}_{o}, r_{e q}\right)} d \vec{r}_{2} p_{\vec{R}_{2}}\left(\vec{r}_{2}\right) \\
& \times w\left(\vec{e}_{o}, r_{e q}, \vec{r}_{1}, \vec{r}_{2}, \theta_{s}, \varphi_{s}\right)
\end{aligned}
$$

where the Monte Carlo weight-function is

$$
\begin{aligned}
w & \left(\vec{e}_{o}, r_{e q}, \vec{r}_{1}, \vec{r}_{2}, \theta_{s}, \varphi_{s}\right) \\
& =\left(\frac{k_{e}}{2 \pi}\right)^{2} \frac{\mathfrak{R}\left(g\left(\vec{r}_{1}, \theta_{s}, \varphi_{s}\right)\right) \Re\left(g\left(\vec{r}_{2}, \theta_{s}, \varphi_{s}\right)\right)+\Im\left(g\left(\vec{r}_{1}, \theta_{s}, \varphi_{s}\right)\right) \Im\left(g\left(\vec{r}_{2}, \theta_{s}, \varphi_{s}\right)\right)}{p_{\vec{R}_{1}}\left(\vec{r}_{1}\right) p_{\vec{R}_{2}}\left(\vec{r}_{2}\right)}
\end{aligned}
$$

with $g$ defined in Eq. (30) and uniform probability density functions $p_{\vec{R}_{1}}\left(\vec{r}_{1}\right)=p_{\vec{R}_{2}}\left(\vec{r}_{2}\right)=1 / P\left(\theta_{0}, r_{e q}\right) \quad$ (arbitrarily chosen as in Section 3.1). In this formulation, the squared modulus was treated by increasing the dimension of the integration domain: instead of integrating over $\mathcal{P}$, we now integrate over $\mathcal{P} \times \mathcal{P}$. In the present context, this is not a drawback since convergence of the Monte Carlo method is dimension-independent $[28,29]$. When compared to the algorithm of Section 3.1 in which only one ray (i.e. one location $\vec{r}$ ) was sampled for each realization of the Monte Carlo algorithm, we hereafter have to sample two rays (i.e. two locations $\vec{r}_{1}$ and $\vec{r}_{2}$ ) for each realization of the particle's orientation and size. This is the only difference between the Monte Carlo algorithms for the cross sections and for the differential cross section. The sampling procedure is the direct translation of Eq. (32):

- Step 1: An orientation $\vec{e}_{o}$ of the particle is sampled over the total solid angle according to the orientation distribution $p_{E_{o}}\left(\vec{e}_{o}\right)$ (any distribution can be used).

- Step 2: A radius $r_{e q}$ for the volume equivalent sphere is sampled over ] $0,+\infty$ [ according to $p_{R_{e q}}\left(r_{e q}\right)$, defining the size of the particle (any distribution can be used).

- Step 3: A first location $\vec{r}_{1}$ is uniformly sampled over the projected surface $\mathcal{P}\left(\theta_{0}, r_{e q}\right)$.

- Step 4: A second location $\vec{r}_{2}$ is uniformly sampled over the projected surface $\mathcal{P}\left(\theta_{0}, r_{e q}\right)$.

- Step 5: The crossing lengths $l\left(\vec{r}_{1}, \vec{e}_{o}, r_{e q}\right)$ and $l\left(\vec{r}_{2}, \vec{e}_{o}, r_{e q}\right)$ of the two rays starting at $\vec{r}_{1}$ and $\vec{r}_{2}$ are computed.

- Step 6: The Monte Carlo weight $w$ is computed according to Eq. (33) (for any value $\varphi_{s}$, e.g. $\varphi=0^{5}$ ).

When implementing the Monte Carlo algorithm corresponding to Eqs. (32) and (33), we record convergence difficulties due to significant oscillations of the weight function (Eq. (33)) with respect to particle orientations $\vec{e}_{o}$. Therefore we implement in Appendix A a variancereduction technique consisting in rearranging the integral formulation (Eq. (32)) in order to explicitly exhibit an analytic solution for the integration over $\varphi_{o}$. In the codes that we provide [32], this optimized Monte Carlo algorithm is implemented to evaluate simultaneously the

\footnotetext{
${ }^{5}$ Note that averaging $W_{s}$ over $\varphi_{o} \in[0,2 \pi]$ is strictly equivalent to averaging it over the scattering angle $\varphi_{s} \in[0,2 \pi]$ (see Appendix A): therefore $W_{s}$ does not depend on $\varphi_{s}$, which means that any $\varphi_{s}$ can be used in $w$ (Eq. (33)).
} 
differential scattering cross-section at several scattering angles $\theta_{s}$, in the same manner as the algorithm of Section 3.1 was used to simultaneously evaluate three cross-sections: several weights $w\left(\theta_{s, 1}\right), w\left(\theta_{s, 2}\right), w\left(\theta_{s, 3}\right)$, etc. are computed at Step 6.

When using radiative properties as input parameters for the resolution of the radiative transfer equation with the Monte Carlo method, as in [23,22], practical implementation requires that the cumulative $C W_{s}\left(\theta_{s}\right)$ of the differential scattering cross-section is available, rather than $W_{s}\left(\theta_{s}\right)$ itself:

$C W_{s}\left(\theta_{s}\right)=2 \pi \int_{0}^{\theta_{s}} \sin \theta^{\prime} W_{s}\left(\theta^{\prime}\right) d \theta^{\prime}$

Since in Eq. (A.13) the scattering angle $\theta_{s}$ only appears in the weight $w\left(\theta_{0}, r_{e q}, \vec{r}_{1}, \vec{r}_{2}, \theta_{s}\right)$, the integral formulation of $C W_{s}\left(\theta_{s}\right)$ is identical to that of $W_{s}\left(\theta_{s}\right)$, replacing only $w$ with the new weight

$w_{C}\left(\theta_{0}, r_{e q}, \vec{r}_{1}, \vec{r}_{2}, \theta_{s}\right)=2 \pi \int_{0}^{\theta_{s}} \sin \theta^{\prime} w\left(\theta_{0}, r_{e q}, \vec{r}_{1}, \vec{r}_{2}, \theta^{\prime}\right) d \theta^{\prime}$

whose analytic expression is given in Appendix B. Therefore, the estimation of $C W_{s}\left(\theta_{S}\right)$ is carried out with the same Monte Carlo algorithm as above, only computing the weight $w_{C}$ instead of $w$ at Step 6. Convergence for $C W_{s}$ is faster than for $W_{s}$, which is particularly advantageous when simulating radiative transfer with the Monte Carlo method.

\subsection{Sensitivity analysis}

When a Monte Carlo algorithm is used for the estimation of a physical quantity $A$, a simple and fast additional procedure can be implemented that simultaneously estimates the partial derivatives $\partial_{\pi} A$ of $A$ with respect to any parameter $\pi[22,26,23]$. A significant benefit of the Monte Carlo integral-formulations that we established in the previous paragraphs is that, based on these formulations, such sensitivity analyses are straightforward in most cases (see [22] for an overview and [26] for the least straightforward case of domain deformation sensitivities). The methodology consists in deriving the integral formulation of $A$ with respect to $\pi$, in order to get an integral formulation for $\partial_{\pi} A$ that is the direct mathematical translation of an algorithm evaluating $\partial_{\pi} A$. Hereafter we focus on the sensitivities of the cross-sections $\sigma_{i}$ with respect to wavelength $\lambda_{e}=2 \pi / k_{e}$, real part $n_{r}$ and complex part $\kappa_{r}$ of the relative refractive index (where $\sigma_{i}$ is either the crosssection for extinction $\sigma_{e x t}$, absorption $\sigma_{a}$ or scattering $\sigma_{s}$ ). We recall the integral formulation (Eq. (24):

$$
\begin{aligned}
\sigma_{i}= & \int_{4 \pi} d \vec{e}_{o} p_{\vec{E}_{o}}\left(\vec{e}_{o}\right) \int_{0}^{\infty} d r_{e q} p_{R_{e q}}\left(r_{e q}\right) \\
& \times \int_{\mathcal{P}\left(\vec{e}_{o}, r_{e q}\right)} d \vec{r} p_{\vec{R}}(\vec{r}) w_{i}\left(\vec{e}_{o}, r_{e q}, \vec{r}, \lambda_{e}, n_{r}, \kappa_{r}\right)
\end{aligned}
$$

where the parameters $\lambda_{e}, n_{r}$ and $\kappa_{r}$ (hereafter noted $\pi$ ) only appear in the weight function $w_{i}$ : they do not appear in the sampling probability density functions, in the integration domain or in any algorithm test. Therefore, the integral formulation of the $n$th derivative $\partial_{\pi}^{n} \sigma_{i}$ is identical to that of $\sigma_{i}$ in Eq. (36), replacing only $w_{i}$ with its derivative $\partial_{\pi}^{n} w_{i}$ (which corresponds to case $a$ in Sec. 2.3 of [22]):

$$
\begin{aligned}
\partial_{\pi}^{n} \sigma_{i}= & \int_{4 \pi} d \vec{e}_{o} p_{\vec{E}_{o}}\left(\vec{e}_{o}\right) \int_{0}^{\infty} d r_{e q} p_{R_{e q}}\left(r_{e q}\right) \\
& \times \int_{\mathcal{P}\left(\vec{e}_{o}, r_{e q}\right)} d \vec{r} p_{\vec{R}^{(}}(\vec{r}) \partial_{\pi}^{n} w_{i}
\end{aligned}
$$

In other words, the same random sampling procedure is used to estimate $\sigma_{i}$ and $\partial_{\pi}^{n} \sigma_{i}$; only the computation of new weight functions $\partial_{\pi}^{n} w_{i}$ is added at Step 5 of the algorithm presented in Section 3.1 (see Fig. 2). The general expressions for these weights $\partial_{\pi}^{n} w_{i}$ are given in Appendix C. Since the calculation of $\sigma_{e x t}, \sigma_{a}, \sigma_{s}$ and their sensitivities to $\lambda_{e}, n_{r}$ and $\kappa_{r}$ are carried out with the same Monte Carlo algorithm, no significant additional CPU time is required for our sensitivity analysis (see discussion in Section 3.1).

The knowledge of these sensitivities can be a significant help in the process of designing databases and interpolating radiative properties within these databases. On one hand it can help to locate regions of the parameter space where a refined mesh is needed due to strong variations of the radiative properties (these are regions where values of $\partial_{\pi}^{2} \sigma_{i}$ are high) and to locate regions where a looser mesh structure is suitable (where values of $\partial_{\pi}^{2} \sigma_{i}$ are lower). On the other hand, once databases including sensitivities are established, an interpolation strategy can be based on the sensitivities: for example, the free parameters in a spline interpolation curve are precisely $\partial_{\pi}^{n} \sigma_{i}$. See kspectrum [41-43] for an example of radiative properties database constructed with such a sensitivity-based approach.

\section{Results for spheroidal and cylindrical photo- synthetic micro-organisms}

The algorithms proposed in Section 3 can be used to address particle shapes with any kind of rotational symmetry, under the condition that the crossing lengths $l$ can be computed. We have implemented the algorithms for spheroids and cylinders (using the analytical expressions of $l$ indicated in Appendices D and E), as a first step towards more complex shapes. The shapes of both the spheroid and the cylinder are defined by their elongation $R$, corresponding to the ratio between the horizontal axis and the axis of revolution $(R<1$ for prolate and $R>1$ for oblate spheroids or cylinders). First, in Section 4.1, we validate Schiff's approximation (and our Monte Carlo implementation) by comparing the results of the algorithms presented in Section 3 with reference results obtained using a T-Matrix code (developed by Mishchenko et al. [3]). Due to the current limitations of T-Matrix codes, such a validation can only be performed for micro-organisms with small elongations (i.e. small values of $|R-1|)$ and small median size-parameters $\bar{x}=2 \pi \bar{r}_{e q} / \lambda_{e}$ (calculated over the size distribution). Here we use $R=0.837$ (typical value for Chlamydomonas reinhardtii $[30,34])$ and $\bar{x}<\bar{x}_{\text {lim }}$, where $\bar{x}_{\text {lim }}$ corresponds to the limit value of the size parameter beyond which the T-Matrix code [3] diverges (here $\bar{x}_{\text {lim }} \approx 36{ }^{6}$ ). Then, in Section 4.2, we apply

\footnotetext{
${ }^{6}$ The limit value $\bar{x}_{\text {lim }}$ of the size parameter beyond which the TMatrix code diverges depends on the choice of the cut-off value $r_{e q, \max }$ for
} 

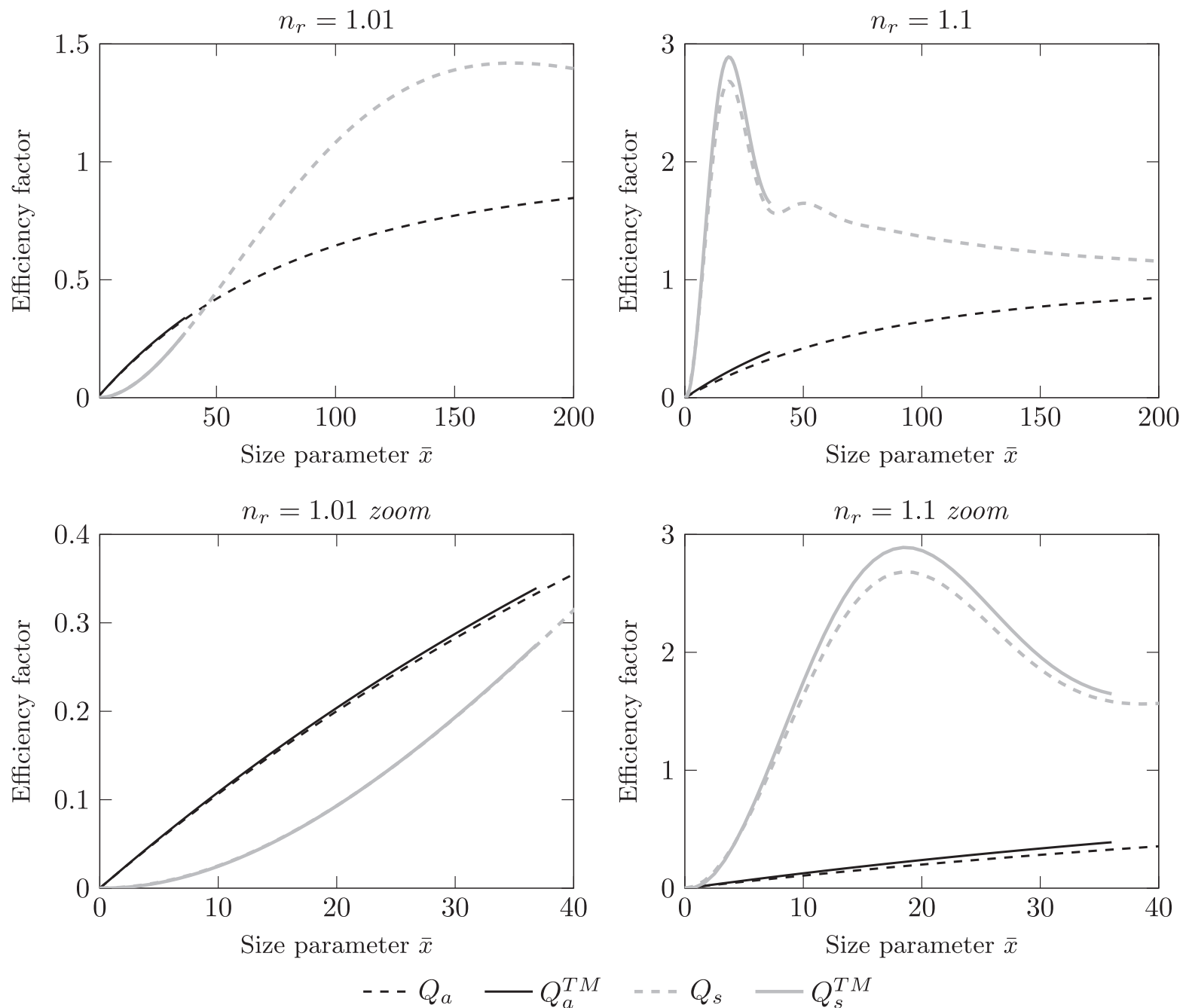

Fig. 3. Absorption $\left(Q_{a}\right)$ and scattering $\left(Q_{s}\right)$ efficiency factors as a function of the (median) particle size parameter $\bar{x}=2 \pi \bar{r}_{e q} / \lambda_{e}$ of a spheroidal particle. Comparison of the predictions of the Schiff's approximation $\left(Q_{i}\right)$ to the reference T-Matrix results ( $\left.Q_{i}\right)$. Parameters: $\lambda_{e}=450 \mathrm{~nm}, s=1.18, R=0.837, \kappa_{r}=4 \cdot 10^{-3}, n_{r}=1.01$ and $n_{r}=1.1$.

Schiff's approximation to larger (up to $\bar{x}=200$ ) and more elongated micro-organisms (up to $R=0.02$, i.e. a particle 50 times longer than wide, typical for Arthrospira platensis). Promising perspectives for assessing the accuracy of Schiff's approximation for such large size parameters and strong elongations include recent advances in methods based on volume discretization such as ADDA [5], experimental measurements in single scattering condition [34] as well as microwave analog measurements [44].

The physical parameter values and orientation/size distributions were chosen in order to represent typical configurations for studies of photosynthetic microorganisms $[34,30]$. More precisely, the following choices were made:

\section{(footnote continued)}

integration over the size distribution $p_{R_{e q}}$ (values of $r_{e q}$ higher than $r_{e q, \max }$ are neglected). Here, $r_{e q, \max }$ was determined to satisfy $p_{R_{e q}}\left(r_{e q}\right)<10^{-3}$ for all $r_{e q}>r_{e q, \max }$.
- the wavelength of the electromagnetic wave propagating in the surrounding medium is $\lambda_{e}=450 \mathrm{~nm}^{7}$;

- the real part of the micro-organism's relative refractive index $n_{r}$ is in the range $[1.01 ; 1.1]$ and its complex part is $\kappa_{r}=4 \cdot 10^{-3}$;

- $p_{\vec{E}}\left(\vec{e}_{o}\right)$ is chosen as an isotropic probability density function, as in [30]:

$p_{\vec{E}_{o}}\left(\vec{e}_{o}\right)=\frac{1}{4 \pi}$

- $p_{R_{e q}}\left(r_{e q}\right)$ is chosen as a log-normal probability density function, as in [30]:

$$
p_{R_{e q}}\left(r_{e q}\right)=\frac{1}{\sqrt{2 \pi} r_{e q} \ln s} \exp \left[-\frac{\left(\ln r_{e q}-\ln \bar{r}_{e q}\right)^{2}}{2 \ln ^{2} s}\right]
$$

\footnotetext{
${ }^{7}$ Here the surrounding medium is water $(n=1.33)$, so the corresponding wavelength in the vacuum is $\lambda_{v}=600 \mathrm{~nm}$, which is within the photosynthetically active spectral range (PAR) [400 nm; $700 \mathrm{~nm}$ ].
} 

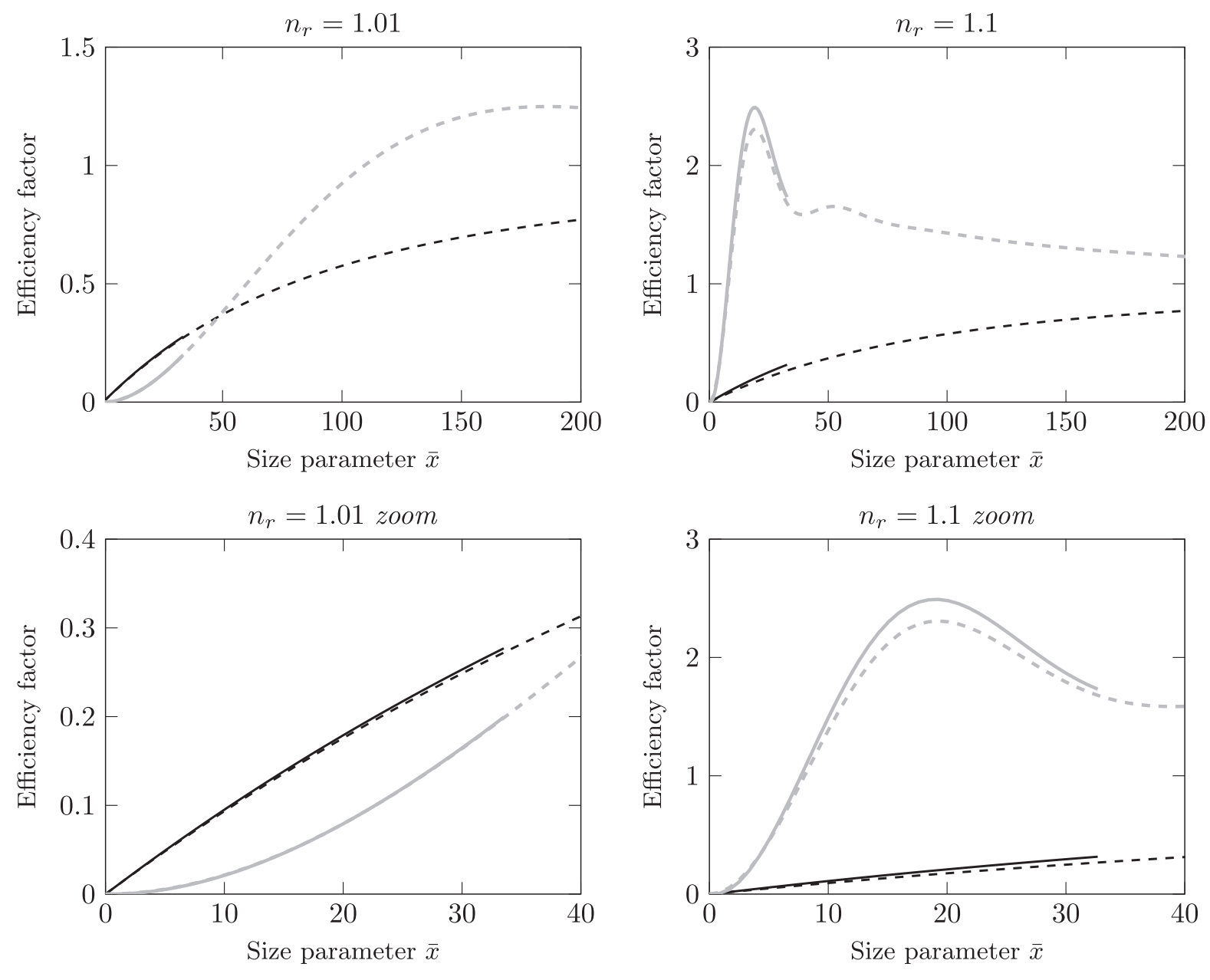

$$
--Q_{a}-Q_{a}^{T M}
$$

$=-Q_{s}-Q_{s}^{T M}$

Fig. 4. Absorption $\left(Q_{a}\right)$ and scattering $\left(Q_{s}\right)$ efficiency factors as a function of the (median) particle size parameter $\bar{x}=2 \pi \bar{r}_{e q} / \lambda_{e}$ of a cylindrical particle. Comparison of the predictions of Schiff's approximation $\left(Q_{i}\right)$ to the reference T-Matrix results $\left(\mathrm{Q}_{i}^{T M}\right)$. Parameters: $\lambda_{e}=450 \mathrm{~nm}, S=1.18, R=0.837, \kappa_{r}=4$. $10^{-3}, n_{r}=1.01$ and $n_{r}=1.1$.
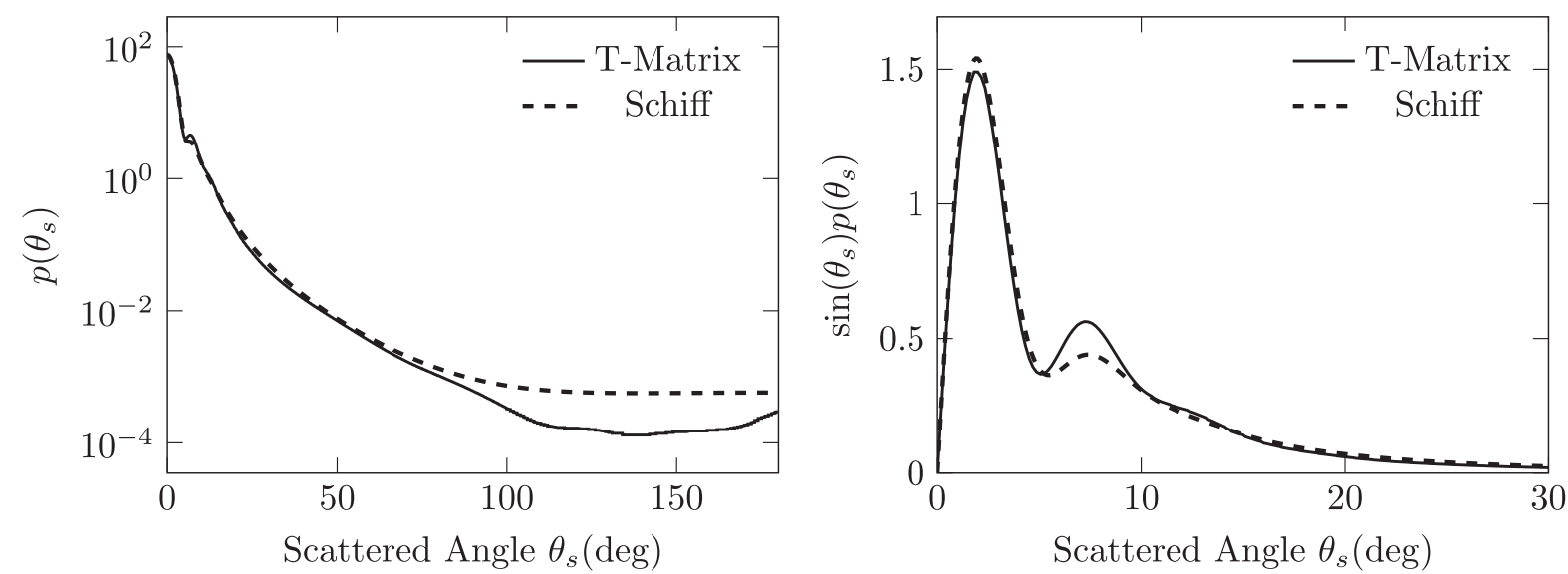

Fig. 5. Single scattering phase function $p\left(\theta_{s}\right)$ as a function of the scattered angle $\theta_{s}$ for a spheroidal particle. Comparison of the predictions of Schiff's approximation to the reference T-Matrix results. Parameters: $\lambda_{e}=450 \mathrm{~nm}, \bar{r}_{e q}=2.55 \mu \mathrm{m}$ (i.e. $\bar{x}=35.6$ ), $s=1.18, R=0.837, \kappa_{r}=4 \cdot 10^{-3}, n_{r}=1.08, \theta_{l}=14^{\circ}$, $A=5.85 \cdot 10^{-14}$ and $B=3.39$.

where $\bar{r}_{e q}$ is the median radius (here $0.07 \mu \mathrm{m} \leq$ $\bar{r}_{e q} \leq 15 \mu \mathrm{m}$, corresponding to $\left.1 \leq \bar{x} \leq 200\right)$ and $s$ is the width parameter of the distribution (here $s=1.18$, typical value for Chlamydomonas reinhardtii).
For graphical reasons, rather than showing directly the cross sections $\sigma_{i}$ and the differential scattering cross sections $W_{s}\left(\theta_{s}\right)$, we present in Figs. 3-6 the corresponding 

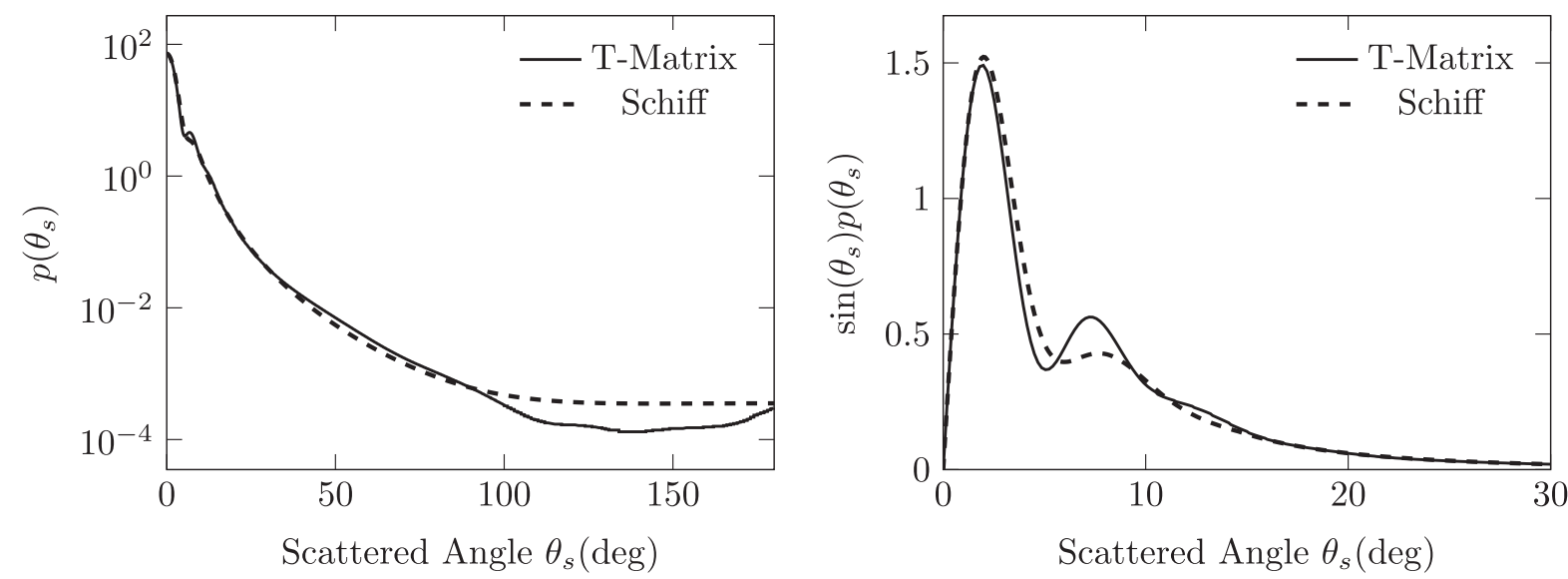

Fig. 6. Single scattering phase function $p\left(\theta_{s}\right)$ as a function of the scattered angle $\theta_{s}$ for a cylindrical particle. Comparison of the predictions of Schiff's approximation to the reference T-Matrix results. Parameters: $\lambda_{e}=450 \mathrm{~nm}, \bar{r}_{e q}=2.38 \mu \mathrm{m}(\mathrm{i} . \mathrm{e} . \bar{x}=33.2), s=1.18, R=0.9, \kappa_{r}=4 \cdot 10^{-3}, n_{r}=1.08, \theta_{l}=15.4^{\circ}$, $A=4.06 \cdot 10^{-14}$ and $B=3.58$.

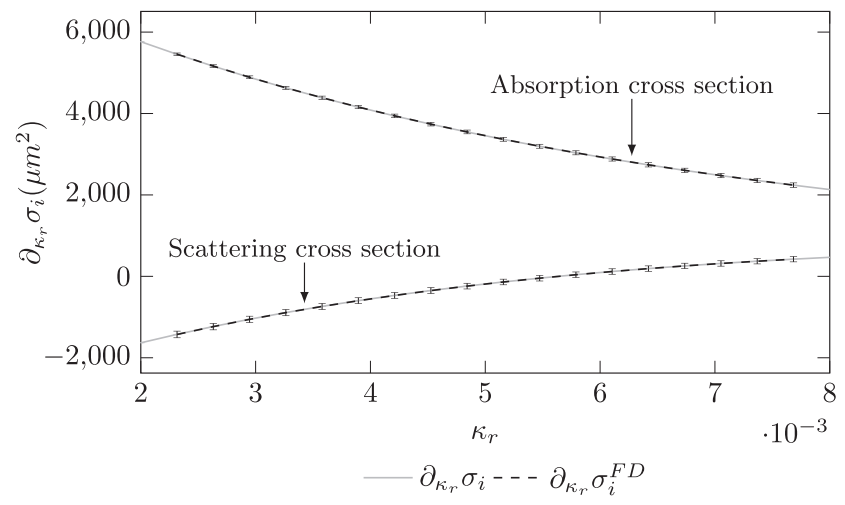

Fig. 7. Sensitivity $\partial_{\kappa_{r}} \sigma_{i}$ of the absorption and scattering cross sections $\sigma_{a}$ and $\sigma_{s}$ to the parameter $\kappa_{r}$ for a spheroidal particle. Comparison of the sensitivities directly computed with the Monte Carlo algorithm $\partial_{\kappa_{r}} \sigma_{i}$ to those obtained by a standard finite difference approach $\partial_{\kappa_{r}} \sigma_{i}^{F D}$. Note that standard deviations for $\partial_{\kappa_{r}} \sigma_{i}$ are negligible compared to those for $\partial_{\kappa_{r}} \sigma_{i}^{F D}$. Parameters: $\kappa_{r} \in\left[2 \cdot 10^{-3} ; 8 \cdot 10^{-3}\right], \lambda_{e}=450 \mathrm{~nm}, n_{r}=1.01, \bar{r}_{e q}=3.963 \mu \mathrm{m}$, $S=1.18, R=0.837$.

efficiency factors

$Q_{i}=\frac{\sigma_{i}}{\left\langle P\left(\vec{e}_{o}, r_{e q}\right)\right\rangle}$,

where $\left\langle P\left(\vec{e}_{o}, r_{e q}\right)\right\rangle$ is the mean area of the projected surface, and the single scattering phase function

$p\left(\theta_{s}\right)=\frac{W_{s}\left(\theta_{s}\right)}{\sigma_{s}}$.

We also show $p\left(\theta_{s}\right) \sin \left(\theta_{s}\right)$ which is proportional to the density of the energy fraction scattered with angle $\theta_{s}$.

\subsection{Validation: comparison of Schiff's approximation with T- Matrix reference results}

Cross sections: Figs. 3 and 4 show the absorption $\left(Q_{a}\right)$ and the scattering $\left(Q_{s}\right)$ efficiencies calculated for, respectively, spheroidal and cylindrical particles with small elongation. The corresponding Monte Carlo simulations were performed with $N=10^{6}$ realizations, which leads to numerical uncertainties always smaller than $0.2 \%$. Comparison of the results obtained using Schiff's approximation to those obtained with the T-Matrix code (in the parameter range still compatible with the T-Matrix code [3] i.e. $\bar{x}<\bar{x}_{\text {lim }}$ ) shows that Schiff's approximation gives highly accurate results for size parameters typical for photosynthetic microorganisms $\left(\bar{x}>10\right.$ corresponding to $r_{e q}>0.7 \mu \mathrm{m}$ ) when the refractive-index contrast is low $\left(n_{r}=1.01\right)$ : the relative difference is always smaller than $2 \%$ (for both $Q_{a}$ and $Q_{s}$, and for both spheroidal and cylindrical particles). As expected, the accuracy progressively decreases when the refractiveindex contrast is increased: for $n_{r}=1.1$ (upper limit value in most studies of photosynthetic micro-organisms), the difference reaches $7.5 \%$ for $Q_{s}$ and $16 \%$ for $Q_{a}$. Moreover, the relative error is (slightly) decreasing with the size parameter $x$ : from $16.1 \%$ at $x=24$ to $15.9 \%$ at $x=36$ for the spheroidal particle in Fig. 3, and from $15.8 \%$ at $x=19.3$ to $15.6 \%$ at $x=32.7$ for the cylindrical particle in Fig. 4 .

Differential scattering cross sections: Figs. 5 and 6 show the single scattering phase function $p\left(\theta_{s}\right)$ (and the quantity $\sin \left(\theta_{s}\right) p\left(\theta_{s}\right)$ that is the contribution of angle $\theta_{s}$ to the cumulative of the phase function, an important quantity in radiative transfer analysis) calculated for, respectively, spheroidal and cylindrical particles. The plots are provided for $n_{r}=1.08$ (which is already a strong refractive-index contrast for photosynthetic micro-organisms). We notice good accuracy up to $\theta_{s} \approx 70^{\circ}$ (Fig. 5) and $\theta_{s} \approx 100^{\circ}$ (Fig. 6). For larger angles, the discrepancy increases, but this contributes very little to the total scattered energy. Overall, the accuracy of Schiff's approximation is still very satisfying with regard to the requirements of photosynthesis engineering.

Sensitivities: The direct calculation of sensitivities of radiative properties to a physical parameter $\pi$ using the Monte Carlo method was validated by comparison with the sensitivities computed using a standard finite difference approach (as in [26]). Here, this is exemplified by the sensitivities of the cross sections $\sigma_{i}$ to the imaginary part $\kappa_{r}$ of the refractive index. On one hand, the direct Monte Carlo sensitivity $\partial_{\kappa_{r}} \sigma_{i}$ at $\kappa_{r}=\kappa_{r, j}$ is estimated using the Monte Carlo weight given in Appendix C (with $N=10^{6}$ realizations). On the other hand, the corresponding finite 
Table 1

Total computation times $t_{0.5 \%}$ (and the corresponding number $N$ of Monte Carlo realizations) required to calculate the three cross sections $\sigma_{i}$ and the cumulative of the differential scattering cross section $C W_{s}\left(\theta_{s}\right)$ of photosynthetic micro-organisms with our Monte Carlo implementation of Schiff's approximation with a relative standard deviation lower than $0.5 \%$ for all $\theta_{s}<\theta_{l}\left(\theta_{l}\right.$ is defined by Eq. (7) with $\tilde{x}$ the small axes of the cylinder/ spheroid). The computation was performed on a computer cluster equipped with 20 Intel(R) Xeon(R) CPU E5-2680 v2 @ $2.80 \mathrm{GHz}$ biprocessors. Parameters: $\lambda_{e}=450 \mathrm{~nm}, \bar{r}_{e q}=7.16 \mu \mathrm{m} \quad$ (i.e. $\quad \bar{x}=100$ ), $s=1.18, \kappa_{r}=4 \cdot 10^{-3}, n_{r}=1.05$.

\begin{tabular}{llll}
\hline Aspect ratio, $R$ & 0.837 & 0.2 & 0.02 \\
\hline Spheroid & & & \\
$\theta_{l}$ & $4.17^{\circ}$ & $5.30^{\circ}$ & $7.77^{\circ}$ \\
$N$ & $4.1 \cdot 10^{6}$ & $1 \cdot 10^{7}$ & $4.5 \cdot 10^{7}$ \\
$t_{0.5 \%}$ using 1 processor & $100 \mathrm{~s}$ & $285 \mathrm{~s}$ & $1694 \mathrm{~s}$ \\
$t_{0.5 \%}$ using 20 processors & $11 \mathrm{~s}$ & $25 \mathrm{~s}$ & $145 \mathrm{~s}$ \\
Cylinder & & & \\
$\theta_{l}$ & $9.0^{\circ}$ & $11.3^{\circ}$ & $16.7^{\circ}$ \\
$N$ & $5.3 \cdot 10^{6}$ & $9.3 \cdot 10^{6}$ & $4 \cdot 10^{7}$ \\
$t_{0.5 \%}$ using 1 processor & $140 \mathrm{~s}$ & $290 \mathrm{~s}$ & $1763 \mathrm{~s}$ \\
$t_{0.5 \%}$ using 20 processors & $14 \mathrm{~s}$ & $26 \mathrm{~s}$ & $146 \mathrm{~s}$ \\
\hline
\end{tabular}

difference sensitivity is computed as

$\left.\partial_{\kappa_{r}} \sigma_{i}^{F D}\right|_{\kappa_{r}=\kappa_{r, j}}=\frac{\sigma_{i}\left(\kappa_{r}+\delta \kappa_{r}\right)-\sigma_{i}\left(\kappa_{r}-\delta \kappa_{r}\right)}{2 \delta \kappa_{r}}$

where $\delta \kappa_{r}$ is small (here $\delta \kappa_{r}=3.2 \cdot 10^{-4}$, note that the finite difference sensitivity converges to the exact sensitivity when $\left.\delta \kappa_{r} \rightarrow 0\right)$, and $\sigma_{i}\left(\kappa_{r}-\delta \kappa_{r}\right)$ and $\sigma_{i}\left(\kappa_{r}+\delta \kappa_{r}\right)$ are estimated with the Monte Carlo algorithm used in Fig. 3. The associated standard deviation is estimated as

$\left.\operatorname{std}\left(\partial_{\kappa_{r}} \sigma_{i}^{F D}\right)\right|_{\kappa_{r}=\kappa_{r, j}} \approx \frac{\operatorname{std}\left(\sigma_{i}\left(\kappa_{r}+\delta \kappa_{r}\right)\right)+\operatorname{std}\left(\sigma_{i}\left(\kappa_{r}-\delta \kappa_{r}\right)\right)}{2 \delta \kappa_{r}}$

where $\operatorname{std}\left(\sigma_{i}\left(\kappa_{r}+\delta \kappa_{r}\right)\right)$ and $\operatorname{std}\left(\sigma_{i}\left(\kappa_{r}-\delta \kappa_{r}\right)\right)$ are the standard deviations for the Monte Carlo estimates of $\sigma_{i}\left(\kappa_{r}-\delta \kappa_{r}\right)$ and $\sigma_{i}\left(\kappa_{r}+\delta \kappa_{r}\right)$.

The corresponding numerical results obtained for a spheroidal particle are shown in Fig. 7 and confirm that the sensitivities are correctly calculated using the Monte Carlo weight of Appendix C.

\subsection{Application to large and elongated photosynthetic micro-organisms}

In [30], we used Schiff's approximation to predict radiative properties required for the resolution of the radiative transfer equation in typical photobioreactor configurations. This involved size parameters up to $\bar{x} \approx 200$ and elongations up to $R \approx 0.02$ (for Arthospira platensis). All the corresponding numerical results are provided in [30], including validation against experimental normal-hemispherical transmittance measurements. In the present paper, Figs. 3 and 4 also display absorption and scattering cross sections of spheroidal and cylindrical particles for large size parameters (up to $\bar{x}=200$ ). We do not show more numeric values of radiative properties, because this is not the objective of the present paper, which focuses on the calculation method. We prefer to provide the computational times required to calculate the three cross sections $\sigma_{i}$ and the cumulative of the differential scattering cross section $C W_{s}\left(\theta_{s}\right)$ (we do not compute $W_{s}\left(\theta_{s}\right)$ as all the information is already contained in its cumulative $\left.C W_{s}\left(\theta_{s}\right)\right)$ at small angles $\left(\theta_{s} \leq \theta_{l}\right)$ for cylindrical and spheroidal particles with large size parameter $(\bar{x}=100)$ and elongations up to $R=0.02$ (which corresponds to typical values for photosynthetic micro-organisms). All these quantities were evaluated with an accuracy of $0.5 \%$ (i.e. all the relative standard deviations $\operatorname{std}\left(\sigma_{i}\right) / \sigma_{i}$ and $\operatorname{std}\left(C W_{s}\left(\theta_{s}\right)\right) / C W_{s}\left(\theta_{s}\right)$ are lower than $0.5 \%)$. Note that the required number $N$ of Monte Carlo realizations (and therefore the total computation time) is governed by $\mathrm{CW}_{s}\left(\theta_{l}\right)$, for which convergence is the lowest. All the computations were carried out on a computer cluster equipped with 20 Intel(R) Xeon(R) CPU E5-2680 v2 @ $2.80 \mathrm{GHz}$ biprocessors. The computational time for the three cross sections $\sigma_{i}$ is always lower than $3 s$ (even when using only a single processor). With regard to the cumulative of the differential scattering cross section, the number of small scattering angles $\theta_{s, k} \leq \theta_{l}, k=1,2,3, \ldots$, at which $C W_{s}\left(\theta_{s, k}\right)$ is computed by the Monte Carlo algorithm is defined so that there are 1000 equally-spaced scattering angles in $[0, \pi]$ : e.g. this corresponds to 93 small scattering angles for $R=0.02$. Note that, in this case, $C W_{s}\left(\theta_{l}\right) / \sigma_{s} \approx 0.97$ i.e. $97 \%$ of the scattered energy is described by the small-angle model. In Table 1 , when using a single processor, we recorded total computational times always lower than $30 \mathrm{~min}$, even for very elongated micro-organisms $(R=0.02)$. With parallelization on 20 processors, the computation time was reduced to less than $3 \mathrm{~min}$. Such computation times are adequate for applications such as the production of databases of radiative properties for radiative transfer studies in photobioreactors (see [45] for a recent example). However, the convergence of the differential scattering cross section $W_{s}\left(\theta_{s}\right)$ itself is much slower: in the case of a cylinder with $R=0.2$, the computational time required to achieve a precision of $0.5 \%$ is ca. $3 \mathrm{~h}$.

\section{Conclusion and perspectives}

In this work, a Monte Carlo implementation of Schiff's approximation for homogeneous cylindrical and spheroidal particles has been developed and applied to the calculation of the radiative properties of photosynthetic micro-organisms with large size parameters and strong elongations, for which the standard numerical methods of rigorously solving Maxwell's equations are still impracticable. The construction of our algorithms has been described and the corresponding codes are available in Supplementary Material [32].

The following conclusions can be drawn:

- Resolution of Schiff's approximation for the differential scattering cross-section (and its cumulative) at small angles has been made possible by increasing the dimension of the integration domain in order to treat the nonlinearity corresponding to the squared modulus of the scattering amplitude. This leads to a double sampling procedure for the location on the projected surface of the particle.

- A comparison of Schiff's approximation with a T-Matrix code [3] (in the parameter range where the T-Matrix code [3] is still practicable) leads to satisfactory quantitative agreement with respect to the requirements of 
photosynthesis engineering. The accuracy of the predictions (and the decrease of the relative error when increasing the size parameter $x$ ) permitted us to gain confidence in the pertinence of Schiff's approximation for estimating the radiative properties of homogeneous, simple-shaped and optically soft particles.

- Calculating the sensitivities of the cross-sections to $\lambda_{e}, n_{r}$ and $\kappa_{r}$ is straightforward, which is useful for the efficient production of radiative-properties databases.

- The CPU time cost is compatible with the production of databases gathering $\sigma_{e x t}, \sigma_{a}, \sigma_{s}$ and the cumulative $C W_{s}$ of the differential scattering cross-section of microorganisms. This is the information required for simulating radiative transfer within photobioreactors with the Monte Carlo method, as in [45]. Nevertheless, the evaluation of the differential scattering cross-section $W_{s}$ itself remains problematic (CPU time ca. three hours per wavelength) due to convergence difficulties, despite the convergence enhancement proposed in Appendix A.

A point worth particular attention in subsequent studies is our simplified model for scattering at large angles that does not correspond to the same level of accuracy than for small angles. We had to introduce it to avoid severe convergence difficulties when solving the original Schiff's approximation for $\theta_{s} \geq \theta_{l}$. This permits us to obtain phase functions that allow radiative transfer simulations with fair accuracy, for applications in which large-angle scattering has small influence only. ${ }^{8}$ Note that, for such applications, our simplified model is a significant improvement when compared to a simple isotropic model (i.e. $W_{s}\left(\theta_{s}\right)=A$ for $\theta_{s} \geq \theta_{l}$ instead of Eq. (18)), see Figs. 5 and 6. Applications that are focused on large-angle scattering (e.g., in oceanography) might motivate future investigations.

The present work focuses on homogeneous particles whose bounding surface can be described by quadratic parametric equations (e.g. cylinder and spheroid), in which case analytical expressions are available for the crossing lengths $l$ of straight rays through the particle (see Fig. 1). Although this is a significant improvement compared with the standard sphere-equivalent description, these quadratic shapes still do not encompass the great morphological diversity of photosynthetic micro-organisms. Indeed, it has been shown in [30] that some complex-shaped micro-algae (e.g. Arthospira platensis) require a finer description of their geometry. When applying Schiff's approximation to such complex (non-quadratic) particles, the main difficulty is that of calculating the crossing lengths $l$ (see [46]). With our Monte Carlo approach this difficulty is reduced to that of calculating intersections between straight rays and the complex particle's bounding surface. In this perspective, we are presently investigating the extension of our codes through the use of recent computation tools issued by the computer-graphics research community for the acceleration of ray-tracing in complex geometries [22]. Another perspective of the

${ }^{8}$ This is the case for photobioreactors, in which typically, more than $90 \%$ of the energy scattered by the microalgae corresponds to angles smaller than the limit angle $\theta_{l}$, and where the relevant radiative quantity is the irradiance field. present work concerns its extension to heterogeneous particles, which can benefit from recent developments concerning integral formulation of null-collision Monte Carlo algorithms [37]. Finally, based on the Monte Carlo integral formulation that is developed in the present paper, further convergence enhancements will be made possible using the zero variance approach $[23,37,38]$.

\section{Acknowledgments}

'This work has been sponsored by the French government research-program "Investissements d'avenir" through the ANR programs PHOTOBIOH2 (2005-08), BIOSOLIS (200811), ALGOH2 (2011-15), PRIAM (2013-15), the IMobS3 and SOLSTICE Laboratories of Excellence (ANR-10-LABX-16-01 and ANR-10-LABX-22-01), by the European Union through the program "Regional competitiveness and employment" 2007-2013 (ERDF Auvergne region), and by the Auvergne region. It is also founded by the CNRS through the PIE program PHOTORAD (2010-11) and the PEPS program "Intensification des transferts radiatifs pour le développement de photobioréacteurs a haute productivité volumique" (20122013). The authors acknowledge Méso-Star SAS [43] for fruitful discussions about the perspectives of this work, concerning radiative properties databases and ray tracing in complex geometry. We also acknowledge the CNRS research federation FedESol where fecund discussions and debates take place every year since 2012 .

\section{Appendix A. Convergence enhancement for the Monte Carlo algorithm evaluating the differential scattering cross section at small angles}

The present Appendix provides a modified Monte Carlo algorithm that addresses the convergence difficulties observed when evaluating the differential scattering crosssection using the algorithm presented in Section 3.2 (corresponding to Eqs. (32) and (33)). This optimized Monte Carlo algorithm evaluates the very same quantity as the original one (no approximation is made and the corresponding integral formulations are mathematically equal), the only difference is the convergence speed. The only restriction of this optimization is that the orientation angle $\varphi_{o}$ of the particle must be uniformly distributed. This algorithm is implemented in the codes that we provide along with the present paper [32].

The observed convergence difficulties are due to significant oscillations of the weight function (Eq. (33)) with respect to particle orientations $\vec{e}_{o}$. Therefore, we propose hereafter to enhance the convergence of this algorithm by implementing the variance-reduction technique called reduction of dimensionality [27], which consists in rearranging the integral formulation (Eq. (32)) in order to explicitly exhibit an analytic (or symbolic) solution for the integration over the dimension responsible for the main variance source. In the present case this is the integration over the particle's orientation

$$
\int_{4 \pi} d \vec{e}_{o} p_{E_{o}}\left(\vec{e}_{o}\right) \equiv \int_{0}^{\pi} d \theta_{o} p_{\Theta_{o}}\left(\theta_{o}\right) \int_{0}^{2 \pi} d \varphi_{o} p_{\Phi_{o}}\left(\varphi_{o}\right)
$$


where the vector $\vec{e}_{o}$ is written as a function of two orientation angles $\theta_{o}$ and $\varphi_{o}$ :

$\vec{e}_{o}=\mid \begin{array}{ll}\sin \left(\theta_{0}\right) & \cos \left(\varphi_{o}\right) \\ \sin \left(\theta_{0}\right) & \sin \left(\varphi_{o}\right) \\ \cos \left(\theta_{0}\right) & \end{array}$

in the coordinate system $(x, y, z)$ of Fig. 1a. More precisely, we show hereafter that integration over $\varphi_{0} \in[0,2 \pi]$ in Eq. (32) is analytic in the case of uniform probability density function $p_{\Phi_{o}}\left(\varphi_{o}\right)=1 / 2 \pi$.

Analytic solution for the integration over $\varphi_{0} \in[0,2 \pi]$ : First of all, one has to notice that averaging $W_{s}$ over $\varphi_{o} \in[0,2 \pi]$ is strictly equivalent to averaging it over the scattering angle $\varphi_{s} \in[0,2 \pi]$ (see Fig. 1a). Indeed, this is the reason why the differential scattering cross-section of randomly oriented particles is a function of $\theta_{s}$ only and not a function of $\theta_{s}$ and $\varphi_{s}$. Therefore, we replace the integration over $\varphi_{o} \in[0,2 \pi]$ in Eq. (A.1) by an integration over $\varphi_{s} \in[0,2 \pi]$ with uniform probability density function $p_{\Phi_{\mathrm{s}}}\left(\varphi_{S}\right)=1 / 2 \pi$ and we address the integral

$$
\begin{aligned}
I= & \int_{0}^{2 \pi} d \varphi_{s} \frac{1}{2 \pi}\left[\Re\left(g\left(\vec{r}_{1}, \theta_{s}, \varphi_{s}\right)\right) \mathfrak{R}\left(g\left(\vec{r}_{2}, \theta_{s}, \varphi_{s}\right)\right)\right. \\
& \left.+\Im\left(g\left(\vec{r}_{1}, \theta_{s}, \varphi_{s}\right)\right) \mathfrak{J}\left(g\left(\vec{r}_{2}, \theta_{s}, \varphi_{s}\right)\right)\right]
\end{aligned}
$$

that appears in Eq. (32) (injecting Eqs. (33) and (A.1)) with $\varphi_{s}$ instead of $\varphi_{o}$ into Eq. (32)). Introducing the functions

$$
\begin{aligned}
& \Gamma\left(\vec{r}, \theta_{s}, \varphi_{s}\right)=k_{e} \theta_{s}\left(x \cos \varphi_{s}+y \sin \varphi_{s}\right) \\
& \beta_{r}(\vec{r})=k_{e}\left(n_{r}-1\right) l\left(\vec{r}, \vec{e}_{o}, r_{e q}\right) \\
& \beta_{i}(\vec{r})=k_{e} \kappa_{r} l\left(\vec{r}, \vec{e}_{o}, r_{e q}\right)
\end{aligned}
$$

the integrand in Eq. (A.3) can be written

$$
\begin{aligned}
& \mathfrak{R}\left(g\left(\vec{r}_{1}, \theta_{s}, \varphi_{s}\right)\right) \mathfrak{R}\left(g\left(\vec{r}_{2}, \theta_{s}, \varphi_{s}\right)\right) \\
& +\Im\left(g\left(\vec{r}_{1}, \theta_{s}, \varphi_{s}\right)\right) \Im\left(g\left(\vec{r}_{2}, \theta_{s}, \varphi_{s}\right)\right)=\cos \left(\Gamma\left(\vec{r}_{1}, \theta_{s}, \varphi_{s}\right)\right. \\
& \left.-\Gamma\left(\vec{r}_{2}, \theta_{s}, \varphi_{s}\right)\right)\left[1+\exp \left[-\left(\beta_{i}\left(\vec{r}_{1}\right)\right.\right.\right. \\
& \left.\left.+\beta_{i}\left(\vec{r}_{2}\right)\right)\right] \cos \left(\beta_{r}\left(\vec{r}_{1}\right)-\beta_{r}\left(\vec{r}_{2}\right)\right) \\
& -\exp \left(-\beta_{i}\left(\vec{r}_{1}\right)\right) \cos \left(\beta_{r}\left(\vec{r}_{1}\right)\right) \\
& \left.-\exp \left(-\beta_{i}\left(\vec{r}_{2}\right)\right) \cos \left(\beta_{r}\left(\vec{r}_{2}\right)\right)\right] \\
& +\sin \left(\Gamma\left(\vec{r}_{1}, \theta_{s}, \varphi_{s}\right)-\Gamma\left(\vec{r}_{2}, \theta_{s}, \varphi_{s}\right)\right) \\
& {\left[\exp \left[-\left(\beta_{i}\left(\vec{r}_{1}\right)+\beta_{i}\left(\vec{r}_{2}\right)\right)\right] \sin \left(\beta_{r}\left(\vec{r}_{1}\right)-\beta_{r}\left(\vec{r}_{2}\right)\right)\right.} \\
& -\exp \left(-\beta_{i}\left(\vec{r}_{1}\right)\right) \sin \left(\beta_{r}\left(\vec{r}_{1}\right)\right)+\exp \left(-\beta_{i}\left(\vec{r}_{2}\right)\right) \\
& \left.\sin \left(\beta_{r}\left(\vec{r}_{2}\right)\right)\right]
\end{aligned}
$$

where the only dependency on $\varphi_{s}$ is within

$\Gamma\left(\vec{r}_{1}, \theta_{s}, \varphi_{s}\right)-\Gamma\left(\vec{r}_{2}, \theta_{s}, \varphi_{s}\right)=k_{e} \theta_{s}\left(\Delta x \cos \varphi_{s}+\Delta y \sin \varphi_{s}\right)$

with $\Delta x=x_{1}-x_{2}$ and $\Delta y=y_{1}-y_{2}$. Then, introducing $|\Delta \vec{r}|=\sqrt{\Delta x^{2}+\Delta y^{2}} \quad$ and $\quad \varphi_{\Delta} \vec{r}=\arctan (-\Delta x / \Delta y) \pm \pi$, Eq. (A.8) is written as

$$
\Gamma\left(\vec{r}_{1}, \theta_{s}, \varphi_{s}\right)-\Gamma\left(\vec{r}_{2}, \theta_{s}, \varphi_{s}\right)=k_{e} \theta_{s}\|\Delta \vec{r}\| \sin \left(\varphi_{s}-\varphi_{\Delta} \vec{r}\right)
$$

Finally, integration over $\varphi_{S}$ is performed by using the substitution $\varphi_{s}^{\prime}=\varphi_{s}-\varphi_{\Delta} \vec{r}$ :

$$
\begin{array}{r}
\int_{0}^{2 \pi} \frac{1}{2 \pi} \sin \left(\Gamma\left(\vec{r}_{1}, \theta_{s}, \varphi_{s}\right)-\Gamma\left(\vec{r}_{2}, \theta_{s}, \varphi_{s}\right)\right) d \varphi_{S} \\
=\int_{0}^{2 \pi} \frac{1}{2 \pi} \sin \left(k_{e} \theta_{s}\|\Delta \vec{r}\| \sin \varphi_{S}^{\prime}\right) d \varphi_{s}^{\prime}=0
\end{array}
$$

and

$$
\begin{aligned}
\int_{0}^{2 \pi} & \frac{1}{2 \pi} \cos \left(\Gamma\left(\vec{r}_{1}, \theta_{s}, \varphi_{s}\right)-\Gamma\left(\vec{r}_{2}, \theta_{s}, \varphi_{s}\right)\right) d \varphi_{s} \\
= & \int_{0}^{2 \pi} \frac{1}{2 \pi} \cos \left(k_{e} \theta_{s}\|\Delta \vec{r}\| \sin \varphi_{s}^{\prime}\right) d \varphi_{s}^{\prime}=J_{0}\left(k_{e} \theta_{s}\|\Delta \vec{r}\|\right)
\end{aligned}
$$

where $J_{0}$ is the Bessel function of the first kind. Therefore, we obtain the following analytic solution for Eq. (A.3):

$$
\begin{aligned}
I= & J_{0}\left(k_{e} \theta_{s}\|\Delta \vec{r}\|\right)\left(1+\exp \left[-\left(\beta_{i}\left(\vec{r}_{1}\right)+\beta_{i}\left(\vec{r}_{2}\right)\right)\right] \cos \left[\beta_{r}\left(\vec{r}_{1}\right)\right.\right. \\
& \left.\left.-\beta_{r}\left(\vec{r}_{2}\right)\right]-\exp \left(-\beta_{i}\left(\vec{r}_{1}\right)\right) \cos \left(\beta_{r}\left(\vec{r}_{1}\right)\right)\right] \\
& \left.-\exp \left(-\beta_{i}\left(\vec{r}_{2}\right)\right) \cos \left(\beta_{r}\left(\vec{r}_{2}\right)\right)\right)
\end{aligned}
$$

Final Monte Carlo integral-formulation: Injecting the analytic solution Eq. (A.12) into Eq. (32), we obtain the integral formulation of our optimized Monte Carlo algorithm for evaluating the differential scattering crosssection at small angles:

$$
\begin{aligned}
& W_{s}\left(\theta_{s}\right)=\int_{0}^{\pi} d \theta_{0} p_{\Theta_{0}}\left(\theta_{0}\right) \int_{0}^{\infty} d r_{e q} p_{R_{e q}}\left(r_{e q}\right) \\
& \quad \times \int_{\mathcal{P}\left(\theta_{0}, r_{e q}\right)} d \vec{r}_{1} p_{\vec{R}_{1}}\left(\vec{r}_{1}\right) \\
& \int_{\mathcal{P}\left(\theta_{0}, r_{e q}\right)} d \vec{r}_{2} p_{\vec{R}_{2}}\left(\vec{r}_{2}\right) w\left(\theta_{0}, r_{e q}, \vec{r}_{1}, \vec{r}_{2}, \theta_{s}\right)
\end{aligned}
$$

where we arbitrarily choose uniform probability density functions $p_{\vec{R}_{1}}\left(\vec{r}_{1}\right)=p_{\vec{R}_{2}}\left(\vec{r}_{2}\right)=1 / P\left(\theta_{0}, r_{e q}\right)$ for the locations $\vec{r}_{1}$ and $\vec{r}_{2}$ (see Section 3.1), and where the weight function is:

$$
\begin{aligned}
w\left(\theta_{0}, r_{e q}, \vec{r}_{1}, \vec{r}_{2}, \theta_{s}\right)= & \left(\frac{k_{e} P\left(\theta_{o}, r_{e q}\right)}{2 \pi}\right)^{2} \\
& \times J_{0}\left(k_{e} \theta_{s}\|\Delta \vec{r}\|\right)\left(1+\exp \left[-\left(\beta_{i}\left(\vec{r}_{1}\right)\right.\right.\right. \\
& \left.\left.+\beta_{i}\left(\vec{r}_{2}\right)\right)\right] \cos \left[\beta_{r}\left(\vec{r}_{1}\right)\right. \\
& \left.\left.-\beta_{r}\left(\vec{r}_{2}\right)\right]-\exp \left(-\beta_{i}\left(\vec{r}_{1}\right)\right) \cos \left(\beta_{r}\left(\vec{r}_{1}\right)\right)\right] \\
& \left.-\exp \left(-\beta_{i}\left(\vec{r}_{2}\right)\right) \cos \left(\beta_{r}\left(\vec{r}_{2}\right)\right)\right)
\end{aligned}
$$

with $\beta_{r}$ and $\beta_{i}$ defined at Eqs. (A.5) and (A.6) respectively (note that $\beta_{r}$ and $\beta_{i}$ only depend on $\vec{r}_{1,2}$ via the crossinglength $\left.l\left(\vec{r}_{1,2}\right)\right)$.

The sampling procedure of the corresponding Monte Carlo algorithm is the same as in Section 3.2, except for that only $\theta_{0}$ is sampled at Step 1 and that the Monte Carlo weight $w$ is computed according to Eq. (A.14) at Step 6. The only restriction of this optimized algorithm is that the orientation angle $\varphi_{o}$ of the particle is uniformly distributed. 


\section{Appendix B. Cumulative}

The purpose of the present Appendix is to derive the analytical expression of the Monte Carlo weight function $w_{C}$ for the cumulative differential scattering cross section $C W_{s}\left(\theta_{S}\right)$ (for small scattering angles i.e. $\theta_{s} \leq \theta_{l}$ ), defined as (see Eq. (35))

$w_{C}\left(\theta_{o}, r_{e q}, \vec{r}_{1}, \vec{r}_{2}, \theta_{s}\right)=2 \pi \int_{0} \sin \theta^{\prime} w\left(\theta_{0}, r_{e q}, \vec{r}_{1}, \vec{r}_{2}, \theta^{\prime}\right) d \theta^{\prime}$

where $w$ (whose expression is given by Eq. (A.14)) depends on the scattering angle $\theta_{s}$ only via the argument of the Bessel function $J_{0}\left(k_{e} \theta_{s}\|\Delta \vec{r}\|\right)$. Therefore, we can focus on

$$
\begin{aligned}
\int_{0}^{\theta_{s}} & \sin \theta J_{0}\left(k_{e} \theta^{\prime}\|\Delta \vec{r}\|\right) d \theta^{\prime} \\
& \approx \int_{0}^{\theta_{s}} \sin \theta J_{0}\left(2 k_{e} \sin \left(\theta^{\prime} / 2\right)\|\Delta \vec{r}\|\right) d \theta^{\prime} \\
& =\int_{0}^{2 \sin \left(\theta_{s} / 2\right)} X J_{0}\left(k_{e} X\|\Delta \vec{r}\|\right) d X \\
& =2 \sin \left(\theta_{s} / 2\right) \frac{J_{1}\left(2 k_{e} \sin \left(\theta_{s} / 2\right)\|\Delta \vec{r}\|\right)}{k_{e}\|\Delta \vec{r}\|} \\
& \approx \theta_{s} \frac{J_{1}\left(k_{e} \theta_{s}\|\Delta \vec{r}\|\right)}{k_{e}\|\Delta \vec{r}\|}
\end{aligned}
$$

where we note that replacing $J_{0}\left(k_{e} \theta^{\prime}\|\Delta \vec{r}\|\right)$ by $J_{0}\left(2 k_{e} \sin \left(\theta^{\prime} / 2\right)\|\Delta \vec{r}\|\right)$ (in the first line of the mathematical derivation) is not an additional approximation, but rather "suppresses" an approximation that was used in [20] to derive Schiff's approximation at small scattering angles. Indeed, in [20] $2 \sin \left(\theta_{s} / 2\right)$ is replaced by its first-order Taylor series approximation $2 \sin \left(\theta_{s} / 2\right) \approx \theta_{s}$ around $\theta_{s}=0$. This leads to the following expression of the Monte Carlo weight function for the cumulative $C W_{s}\left(\theta_{s}\right)$ :

$$
\begin{aligned}
w_{C} & \left(\theta_{0}, r_{e q}, \vec{r}_{1}, \vec{r}_{2}, \theta_{s}\right) \\
= & \left.2 \pi \frac{k_{e} P\left(\vec{e}_{o}, r_{e q}\right)}{2 \pi}\right)^{2} \theta_{s} \frac{J_{1}\left(k_{e} \theta_{s}\|\Delta \vec{r}\|\right)}{k_{e}\|\Delta \vec{r}\|} \\
& \times\left[1+\exp \left[-\left(\beta_{i}\left(\vec{r}_{1}\right)+\beta_{i}\left(\vec{r}_{2}\right)\right)\right] \cos \left[\beta_{r}\left(\vec{r}_{1}\right)-\beta_{r}\left(\vec{r}_{2}\right)\right]\right. \\
& \left.-\exp \left(-\beta_{i}\left(\vec{r}_{1}\right)\right) \cos \left(\beta_{r}\left(\vec{r}_{1}\right)\right)\right]-\exp \left(-\beta_{i}\left(\vec{r}_{2}\right) \cos \left(\beta_{r}\left(\vec{r}_{2}\right)\right)\right]
\end{aligned}
$$

Let us recall that the cumulative differential scattering cross section $C W_{s}\left(\theta_{s}\right)$ corresponds to the Monte Carlo integral formulation of Eq. (A.13), replacing only $w$ with $w_{C}$. Therefore, the same algorithm can be used to evaluate both $W_{s}$ and $C W_{s}$ simultaneously (see Appendices D and E).

\section{Appendix C. Sensitivities}

This Appendix provides the analytic expressions of the Monte Carlo weight functions $\partial_{\pi}^{n} w_{i}$ for the sensitivities of the cross sections $\sigma_{i}$ (where $\sigma_{i}$ is either the cross-section for extinction $\sigma_{e x t}$, absorption $\sigma_{a}$ or scattering $\sigma_{s}$ ) with respect to wavelength $\lambda_{e}=2 \pi / k_{e}$, real part $n_{r}$ and complex part $\kappa_{r}$ of the relative refractive index, that appear in Eq. (37).
1. Monte Carlo weight functions for the sensitivities with respect to $\kappa_{r}$ :

$$
\partial_{\kappa_{r}}^{n} W_{e x t}=2 P\left(\vec{e}_{o}, r_{e q}\right)(-1)^{n+1}\left(k_{e} l\right)^{n} e^{-k_{e} \kappa_{r} l} \cos \left(k_{e}\left(n_{r}-1\right) l\right)
$$

$$
\begin{aligned}
& \partial_{\kappa_{r}}^{n} w_{a}=P\left(\vec{e}_{o}, r_{e q}\right)(-1)^{n+1}\left(2 k_{e} l\right)^{n} e^{-2 k_{e} \kappa_{r} l} \\
& \partial_{\kappa_{r}}^{n} w_{s}=\partial_{\kappa_{r}}^{n} w_{e x t}-\partial_{\kappa_{r}}^{n} w_{a}
\end{aligned}
$$

where $l \equiv l\left(\vec{r}, \vec{e}_{o}, r_{e q}\right)$ is the crossing length.

2. Monte Carlo weight functions for the sensitivities with respect to $n_{r}$ :

$$
\begin{aligned}
\partial_{n_{r}}^{n} w_{\text {ext }}= & 2 P\left(\vec{e}_{o}, r_{e q}\right)\left(k_{e} l\right)^{n} e^{-k_{e} k_{r} l} \\
& \times \begin{cases}(-1)^{q+1} \cos \left(k_{e}\left(n_{r}-1\right) l\right) & \text { if } n \text { is odd, } n=2 q \\
(-1)^{p} \sin \left(k_{e}\left(n_{r}-1\right) l\right) & \text { if } n \text { is even, } n=2 p+1\end{cases}
\end{aligned}
$$

$$
\partial_{n_{r}}^{n} w_{a}=0
$$

$$
\partial_{\kappa_{r}}^{n} w_{s}=\partial_{\kappa_{r}}^{n} w_{e x t}-\partial_{\kappa_{r}}^{n} w_{a}
$$

3. Monte Carlo weight functions for the sensitivities with respect to wavelength $\lambda_{e}$ :

$$
\begin{aligned}
& \partial_{\lambda_{e}}^{n} w_{e x t}=2 P\left(\theta_{0}, r_{e q}\right)(-1)^{n} \frac{1}{\lambda_{e}^{2 n}} e^{-\gamma / \lambda_{e}}\left[P_{n}\left(\lambda_{e}\right) \cos \frac{\delta}{\lambda_{e}}\right. \\
& \left.+Q_{n}\left(\lambda_{e}\right) \sin \frac{\delta}{\lambda_{e}}\right] \\
& \partial_{\lambda_{e}}^{n} w_{a}=P\left(\vec{e}_{o}, r_{\text {eq }}\right) e^{-\alpha / \lambda_{e}} \sum_{i=1}^{n} \frac{\beta_{n, i}}{\lambda_{e}^{n+i} \alpha^{i}} \\
& \partial_{\kappa_{r}}^{n} w_{s}=\partial_{\kappa_{r}}^{n} w_{e x t}-\partial_{\kappa_{r}}^{n} w_{a}
\end{aligned}
$$

where $\gamma=2 \pi \kappa_{r} l, \delta=2 \pi\left(n_{r}-1\right) l$ and the quantities $P_{n}$ and $Q_{n}$ are defined by the polynomials sequences

$$
\begin{cases}\forall n \geq 2 & \left\{\begin{array}{l}
\left.P_{n}\left(\lambda_{e}\right)=\left(2(n-1) \lambda_{e}-\gamma\right)\right) P_{n-1}\left(\lambda_{e}\right)-\lambda_{e}^{2} P_{n-1}^{\prime}\left(\lambda_{e}\right)+\delta Q_{n-1}\left(\lambda_{e}\right) \\
\left.Q_{n}\left(\lambda_{e}\right)=\left(2(n-1) \lambda_{e}-\gamma\right)\right) Q_{n-1}\left(\lambda_{e}\right)-\lambda_{e}^{2} Q_{n-1}^{\prime}\left(\lambda_{e}\right)-\delta P_{n-1}\left(\lambda_{e}\right)
\end{array}\right. \\
n=1 & \left\{\begin{array}{l}
P_{1}\left(\lambda_{e}\right)=\gamma \\
Q_{1}\left(\lambda_{e}\right)=\delta
\end{array}\right.\end{cases}
$$

and where $\alpha=4 \pi \kappa_{r} l$ and $\beta_{n, i}$ is defined by

$$
\forall n \in \mathbb{N}^{*}\left\{\begin{array}{l}
\beta_{n, n}=-1 \\
\beta_{n, 1}=(-1)^{n} n ! \\
\beta_{n, i}=-(i+n-1) \beta_{n-1, i}+\beta_{n-1, i-1} \quad \forall i \in[2, n-1]
\end{array}\right.
$$

\section{Appendix D. Detailed monte carlo algorithm for spheroids}

In the present Appendix we give the procedure for sampling locations on the projected surface of a spheroid as well as the expression of the crossing-lengths. The algorithms derived hereafter are implemented in the codes available in [32].

Let us first focus on the integral formulation (Eq. (24)) for the cross-sections $\sigma_{i}$, where we write the orientation vector $\vec{e}_{o}$ as a function of two orientation angles $\theta_{o}$ and $\varphi_{o}$ (injecting Eq. (A.1) into Eq. (24), with $\theta_{o}$ and $\varphi_{o}$ defined in 
Eq. (A.2) and Fig. 1). Then, we notice that the expression of $\sigma_{i}$ is independent of $\varphi_{o}$ and therefore, the average over $\varphi_{o} \in[0,2 \pi]$ vanishes in Eq. (24) that becomes:

$$
\begin{aligned}
\sigma_{i}= & \int_{0}^{\pi} d \theta_{o} p_{\Theta_{o}}\left(\theta_{o}\right) \int_{0}^{\infty} d r_{e q} p_{R_{e q}}\left(r_{e q}\right) \\
& \times \int_{\mathcal{P}\left(\theta_{o}, r_{e q}\right)} d r p_{\vec{R}}(\vec{r}) w_{i}\left(\theta_{o}, r_{e q}, \vec{r}\right)
\end{aligned}
$$

where $w_{i}$ is the Monte Carlo weight-function given in Eqs. (25)-(27). For the differential scattering cross-section at small angles, we apply hereafter the optimized Monte Carlo algorithm presented in Eq. (A.13) (that is restricted to uniformly distributed orientation angles $\varphi_{o}$ ). Again, the integral formulation is independent of $\varphi_{o}$. Therefore, any orientation $\varphi_{o}$ can be arbitrarily chosen in the following (results are independent of $\varphi_{o}$ ) and we choose $\varphi_{o}=0$, that is to say that, in the coordinate system of Fig. 1, the axis of revolution of the spheroid is contained in the plane $(x, y=0, z)$ and the axis $y$ is perpendicular to the axis of revolution.

Here we consider a spheroid with semi-axis $a$ in the direction perpendicular to the axis of revolution and aspect ratio $R$. The semi-axis of revolution of the spheroid is $a / R$. The volume of the spheroid is $V_{p}=(4 \pi / 3) a^{3} / R=(4 \pi / 3) r_{e q}^{3}$, where $r_{e q}$ is the radius of the volume-equivalent sphere, and therefore $a=R^{1 / 3} r_{e q}$. The center of the spheroid is located at $(x=0, y=0, z=0)$ in the coordinate system of Fig. 1. For such a spheroid (see [40]):

- The projected surface $\mathcal{P}$ is an ellipse with semi-axes $a$ along $y$ and semi-axis $a \alpha\left(\theta_{0}\right)$ along $x$, where

$$
\alpha\left(\theta_{0}\right)=\sqrt{\cos ^{2}\left(\theta_{0}\right)+\frac{1}{R^{2}} \sin ^{2}\left(\theta_{0}\right)}
$$

- The area of $\mathcal{P}$ is

$$
P=\pi a^{2} \alpha\left(\theta_{0}\right)
$$

- The crossing length is

$$
l(x, y)=\frac{2 a}{R \alpha\left(\theta_{0}\right)} \sqrt{1-\frac{x^{2}}{a^{2} \alpha\left(\theta_{0}\right)^{2}}-\frac{y^{2}}{a^{2}}}
$$

Integration over the projected surface: We introduce the substitution $x=a \alpha\left(\theta_{0}\right) \rho \cos \varphi$ and $y=a \rho \sin \varphi$ where $(\rho, \varphi)$ is a polar coordinate system associated with the unit disc on the plane $(x, y): \rho \in[0,1]$ and $\varphi \in[0,2 \pi]$. Integration over the projected surface $\mathcal{P}$ becomes

$$
\begin{aligned}
\int_{\mathcal{P}} d r p_{\vec{R}}(\vec{r}) & \equiv \iint_{\mathcal{P}\left(\theta_{o}, r_{e q}\right)} d x d y p_{\vec{R}}(\vec{r}) \\
& \equiv \int_{0}^{2 \pi} d \rho \int_{0}^{1} d \rho a^{2} \alpha\left(\theta_{0}\right) \rho p_{\vec{R}}(\vec{r})
\end{aligned}
$$

and the crossing-length Eq. (D.4) becomes

$l(\rho)=\frac{2 a}{R \alpha\left(\theta_{0}\right)} \sqrt{1-\rho^{2}}$

Then, injecting $p_{\vec{R}}(\vec{r})=1 / P=1 /\left(\pi a^{2} \alpha\left(\theta_{0}\right)\right)$ (uniform sampling over $\mathcal{P}$, see Eq. (D.3)) in Eq. (D.5) and introducing the probability density functions $p_{\Phi}(\varphi)=1 / 2 \pi$ and $p_{R}(\rho)=2 \rho$ (corresponding to uniform sampling of locations on the unit disk) by multiplying and dividing Eq. (D.5) by $p_{\Phi}(\varphi)$ and $p_{R}(\rho)$, we obtain

$\int_{\mathcal{P}} d r p_{\vec{R}}(\vec{r}) \equiv \int_{0}^{2 \pi} d \varphi p_{\Phi}(\varphi) \int_{0}^{1} d \rho p_{R}(\rho)$

where we used $a^{2} \alpha\left(\theta_{0}\right) \rho /\left(\pi a^{2} \alpha\left(\theta_{0}\right) p_{\Phi}(\varphi) p_{R}(\rho)\right)=1$. Therefore, the sampling procedure for the locations on $\mathcal{P}$ implies to sample an angle $\varphi$ according to $p_{\Phi}$ and a distance $\rho$ from $(x=0, y=0)$ according to $p_{R}$.

Since in Eq. (24) the location $\vec{r}$ on the projected surface only appears within the crossing length and since the crossing-length in Eq. (D.6) is not a function of $\varphi$, the integral formulation Eq. (D.1) is independent of $\varphi$. Injecting Eq. (D.7) into Eq. (D.1) and using $\int_{0}^{2 \pi} d \varphi p_{\Phi}(\varphi)=1$, we obtain:

$$
\begin{aligned}
\sigma_{i}= & \int_{0}^{\pi} d \theta_{o} p_{\Theta_{o}}\left(\theta_{o}\right) \int_{0}^{\infty} d r_{e q} p_{R_{e q}}\left(r_{e q}\right) \\
& \times \int_{0}^{1} d \rho p_{R}(\rho) w_{i}\left(\theta_{o}, r_{e q}, \vec{r}\right)
\end{aligned}
$$

Therefore, the sampling procedure of the Monte Carlo algorithm evaluating the cross sections for isotropically oriented spheroids with log-normal size distribution is:

- Step 1: An orientation angle $\theta_{o}$ is sampled over $[0, \pi]$ according to the isotropic probability density function $p_{\Theta_{0}}\left(\theta_{0}\right)=\sin \theta_{0} / 2$.

- Step 2: A radius $r_{e q}$ for the volume equivalent sphere is

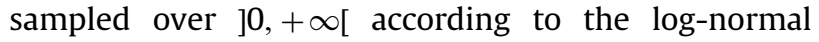
distribution $p_{R_{e q}}\left(r_{e q}\right)$ given in Eq. (39) and the semiaxis $a$ of the spheroid is computed as $a=R^{1 / 3} r_{e q}$.

- Step 3: $\rho$ is sampled over $[0,1]$ according to $p_{P}(\rho)=2 \rho$.

- Step 4: The crossing length $l$ is computed according to Eq. (D.6).

- Step 5: The Monte Carlo weights $w_{\text {ext }}, w_{a}$ and $w_{s}$ are computed according to Eqs. (25)-(27) with $P\left(\vec{e}_{o}, r_{e q}\right)=\pi a^{2} \alpha\left(\theta_{0}\right)$, and the weights for the sensitivities are computed as given in Appendix C.

Applying the same approach for the computation of the differential scattering cross section (see Eq. (A.13)), we obtain the following integral formulation:

$$
\begin{aligned}
W_{s}\left(\theta_{s}\right)= & \int_{0}^{\pi} d \theta_{o} p_{\Theta_{o}}\left(\theta_{o}\right) \int_{0}^{\infty} d r_{e q} p_{R_{e q}}\left(r_{e q}\right) \\
& \times \int_{0}^{2 \pi} d \rho_{1} p_{\Phi_{1}}\left(\varphi_{1}\right) \int_{0}^{1} d \rho_{1} p_{R_{1}}\left(\rho_{1}\right) \int_{0}^{2 \pi} d \rho_{2} p_{\Phi_{2}}\left(\varphi_{2}\right) \\
& \int_{0}^{1} d \rho_{2} p_{R_{2}}\left(\rho_{2}\right) w\left(\theta_{s}\right)
\end{aligned}
$$

where, unlike for $\sigma_{i}$, the integrals over the angles $\varphi_{1}$ and $\varphi_{2}$ are kept since the Monte Carlo weight $w\left(\theta_{s}\right)$ in Eq. (A.14) is a function of the coordinates $x_{1}, x_{2}, y_{1}$ and $y_{2}$ that depend on $\varphi_{1}$ and $\varphi_{2}$. Therefore, the sampling procedure of the Monte Carlo algorithm evaluating the differential scattering cross-section at small angles for isotropically oriented spheroids with log-normal size distribution is:

- Step 1: An orientation angle $\theta_{o}$ is sampled over $[0, \pi]$ according to the isotropic probability density function $p_{\Theta_{0}}\left(\theta_{0}\right)=\sin \theta_{0} / 2$.

- Step 2: A radius $r_{e q}$ for the volume equivalent sphere is sampled over $] 0,+\infty$ [ according to the log-normal distribution $p_{R_{e q}}\left(r_{e q}\right)$ given in Eq. (39) and $a=R^{1 / 3} r_{e q}$ is computed. 
- Step 3: $\varphi_{1}$ is uniformly sampled over $[0,2 \pi]$ and $\rho_{1}$ is sampled over $[0,1]$, defining a first location $\left(x_{1}, y_{1}\right)$ on the projected surface.

- Step 4: The first crossing length $l_{1}\left(\theta_{0}, r_{e q}, \rho_{1}\right)$ is computed according to Eq. (D.6).

- Step 5: $\varphi_{2}$ is uniformly sampled over $[0,2 \pi]$ and $\rho_{2}$ is sampled over $[0,1]$, defining a second location $\left(x_{2}, y_{2}\right)$ on the projected surface.

- Step 6: The second crossing length $l_{2}\left(\theta_{0}, r_{e q}, \rho_{2}\right)$ is computed according to Eq. (D.6).

- Step 7: The Monte Carlo weights $w\left(\theta_{s, 1}\right), w\left(\theta_{s, 2}\right), w\left(\theta_{s, 3}\right)$, etc. for different scattering angles $\theta_{s, 1}, \theta_{s, 2}, \theta_{s, 3}$, etc. are computed according to Eq. (A.14), and the weights $w_{C}\left(\theta_{s, 1}\right), w_{C}\left(\theta_{s, 2}\right), w_{C}\left(\theta_{s, 3}\right)$, etc. for the cumulative of $W_{s}$ are computed according to Eq. (B.3).

\section{Appendix E. Detailed monte carlo algorithm for cylinders}

In the present Appendix we give the procedure for sampling locations on the projected surface of a cylinder as well as the expression of the crossing-lengths. The algorithms derived hereafter are implemented in the codes available in [32].

As for spheroids in Appendix D, any orientation $\varphi_{o}$ can be arbitrarily chosen in the following (results are independent of $\varphi_{o}$ ) and we choose $\varphi_{o}=0$, that is to say that, in the coordinate system of Fig. 1, the axis of revolution of the cylinder is contained in the plane $(x, y=0, z)$ and the axis $y$ is perpendicular to the axis of revolution.

Here we consider a cylinder with radius $a$ and aspect ratio $R$. The length of the cylinder is $2 a / R$. The volume of the cylinder is $V_{p}=2 \pi a^{3} / R=(4 \pi / 3) r_{e q}^{3}$, where $r_{e q}$ is the radius of the volumeequivalent sphere, and therefore $a=(2 / 3 R)^{1 / 3} r_{\text {eq }}$. The center of the cylinder is located at $(x=0, y=0, z=0)$ in the coordinate system of Fig. 1 . In the following, we only treat orientation angles $\theta_{0} \in[0, \pi / 2]$ since, considering the symmetry of the cylinder, the rest $\left(\theta_{0} \in[\pi / 2, \pi]\right)$ gives the same results. For such a cylinder (see [40]):

- The projected surface $\mathcal{P}$ is a rectangle $x \in[-a / R$ $\left.\sin \theta_{o}, a / R \sin \theta_{0}\right], y \in[-a, a]$ with, in addition, two half ellipses centered at $\left(x=-a / R \sin \theta_{0}, y=0\right)$ and $\left(x=a / R \sin \theta_{0}, y=0\right)$ with semi-axes $a$ along $y$ and semi-axis $a \cos \left(\theta_{0}\right)$ along $x$.

- The area of $\mathcal{P}$ is

$P=4 a^{2} \cos \left(\theta_{0}\right)\left(\beta\left(\theta_{0}\right)+\pi / 4\right)$

where $\beta=\tan \theta_{0} / R$

- Introducing $\xi=x /\left(a \cos \theta_{0}\right)$ and $\eta=a / y$, the crossing length is: For $\beta \geq \sqrt{1-\eta^{2}}$,

$$
l / a= \begin{cases}\frac{\xi+\beta+\sqrt{1-\eta^{2}}}{\sin \theta_{o}} & \text { if }|\xi+\beta| \leq \sqrt{1-\eta^{2}} \\ \frac{-\xi+\beta+\sqrt{1-\eta^{2}}}{\sin \theta_{o}} & \text { if }|\xi-\beta| \leq \sqrt{1-\eta^{2}} \\ \frac{2 \sqrt{1-\eta^{2}}}{\sin \theta_{o}} & \text { otherwise }\end{cases}
$$

$$
\begin{aligned}
& \text { For } \beta<\sqrt{1-\eta^{2}}, \\
& \qquad / / a= \begin{cases}\frac{\xi+\beta+\sqrt{1-\eta^{2}}}{\sin \theta_{o}} & \text { if }-\sqrt{1-\eta^{2}}-\beta \leq \xi \leq-\sqrt{1-\eta^{2}}+\beta \\
\frac{2}{R \cos \theta_{o}} & \text { if }-\sqrt{1-\eta^{2}}+\beta \leq \xi \leq \sqrt{1-\eta^{2}}-\beta \\
\frac{-\xi+\beta+\sqrt{1-\eta^{2}}}{\sin \theta_{o}} & \text { if } \sqrt{1-\eta^{2}}-\beta \leq \xi \leq \sqrt{1-\eta^{2}}+\beta \\
\frac{2 \sqrt{1-\eta^{2}}}{\sin \theta_{o}} & \text { otherwise }\end{cases}
\end{aligned}
$$

Integration over the projected surface: Hereafter we use the substitution $x=a \cos \left(\theta_{0}\right) \xi$ and $y=a \eta$ where $(\xi, \eta)$ form a set of unitless coordinates for the projected surface of the cylinder: $y \in[-a, a]$ and $\xi \in\left[-\beta-\sqrt{1-\eta^{2}}, \beta+\sqrt{1-\eta^{2}}\right]$ (see [40]). Integration over the projected surface $\mathcal{P}$ becomes

$$
\begin{aligned}
\int_{\mathcal{P}} d r p_{\vec{R}}(\vec{r}) & \equiv \iint_{\mathcal{P}\left(\theta_{0}, r_{\text {eq }}\right)} d x d y p_{\vec{R}}(\vec{r}) \\
& \equiv \int_{-1}^{1} d \eta \int_{-\left(\beta+\sqrt{1-\eta^{2}}\right)}^{\beta+\sqrt{1-\eta^{2}}} d \xi a^{2} \cos \theta_{o} p_{\vec{R}}(\vec{r})
\end{aligned}
$$

Then, injecting $p_{\vec{R}}(\vec{r})=\frac{1}{P}=1 /\left(4 a^{2} \cos \left(\theta_{0}\right)\left(\beta\left(\theta_{0}\right)+\pi / 4\right)\right)$ (uniform sampling over $\mathcal{P}$, see Eq. (E.1)) in Eq. (D.5) and introducing the uniform probability density functions $p_{H}(\eta)=\frac{1}{2}$ and $p_{\Xi}(\xi \mid \eta)=1 / 2\left(\beta+\sqrt{1-\eta^{2}}\right)$ by multiplying and dividing Eq. (D.5) by $p_{H}(\eta)$ and $p_{\Xi}(\xi \mid \eta)$, we obtain

$$
\int_{\mathcal{P}} d r p_{\vec{R}}(\vec{r}) \equiv \int_{-1}^{1} d \eta p_{H}(\eta) \int_{-\left(\beta+\sqrt{1-\eta^{2}}\right)}^{\beta+\sqrt{1-\eta^{2}}} d \xi p_{\Xi}(\xi \mid \eta) \frac{\beta+\sqrt{1-\eta^{2}}}{\beta+\pi / 4}
$$

where we used $a^{2} \cos \left(\theta_{0}\right) /\left(4 a^{2} \cos \left(\theta_{0}\right)\left(\beta\left(\theta_{0}\right)+\pi / 4\right)\right.$ $\left.p_{H}(\eta) p_{\Xi}(\xi \mid \eta)\right)=\left(\beta+\sqrt{1-\eta^{2}}\right) /(\beta+\pi / 4)$. Therefore, the sampling procedure for the locations on $\mathcal{P}$ implies to sample $\eta$ according to $p_{H}$ and $\xi$ according to $p_{\Xi}$, and the weights have to be multiplied by $\left(\beta+\sqrt{1-\eta^{2}}\right) /(\beta+\pi / 4)$. For the cross sections this leads to:

$w_{i}^{c y l}=w_{i} \frac{\beta+\sqrt{1-\eta^{2}}}{\beta+\pi / 4}$

where $w_{i}$ are given in Eqs. (25)-(27) for the cross sections and in Appendix $C$ for the sensitivities. For the differential scattering cross-section, this procedure has to be repeated twice (first with $x_{1}=a \cos \left(\theta_{0}\right) \xi_{1}, y_{1}=a \eta_{1}$ and then with $\left.x_{2}=a \cos \left(\theta_{0}\right) \xi_{2}, y_{2}=a \eta_{2}\right)$ since Eq. (A.13) includes two integrals over the locations $\left(x_{1}, y_{1}\right)$ and $\left(x_{2}, y_{2}\right)$ on the projected surface. Therefore, the weights become

$w^{c y l}\left(\theta_{s}\right)=w\left(\theta_{s}\right) \frac{\left(\beta+\sqrt{1-\eta_{1}^{2}}\right)\left(\beta+\sqrt{1-\eta_{2}^{2}}\right)}{(\beta+\pi / 4)^{2}}$

where $w\left(\theta_{s}\right)$ is given in Eq. (A.14) for the differential scattering cross-section, and in Appendix $B$ for its cumulative. 
Therefore, the sampling procedure of the Monte Carlo algorithm evaluating the cross sections for isotropically oriented cylinders with log-normal size distribution is:

- Step 1: An orientation angle $\theta_{o}$ is sampled over $[0, \pi / 2]$ according to the isotropic probability density function $p_{\Theta_{0}}\left(\theta_{0}\right)=\sin \theta_{0} \quad$ (we only treat orientation angles $\theta_{0} \in[0, \pi / 2]$ since, considering the symmetry of the cylinder, $\theta_{0} \in[\pi / 2, \pi]$ gives the same results).

- Step 2: A radius $r_{e q}$ for the volume equivalent sphere is sampled over ]0, $+\infty$ [ according to the log-normal distribution $p_{R_{e q}}\left(r_{e q}\right)$ given in Eq. (39) and the radius $a$ of the cylinder is computed as $a=(2 / 3 R)^{1 / 3} r_{e q}$.

- Step 3: $\eta$ is uniformly sampled over $[-1,1]$ and $\xi$ is uniformly sampled over $\left[-\beta\left(\theta_{0}\right)-\sqrt{1-\eta^{2}}, \beta\left(\theta_{0}\right)+\sqrt{1-\eta^{2}}\right]$, defining a location $(x, y)$ on the projected surface.

- Step 4: The crossing length $l$ is computed according to Eqs. (E.2) and (E.3).

- Step 5: The Monte Carlo weights $w_{e x t}, w_{a}$ and $w_{s}$ are computed according to Eq. (E.6).

The sampling procedure of the Monte Carlo algorithm evaluating the differential scattering cross-section at small angles for isotropically oriented cylinders with log-normal size distribution is:

- Step 1: An orientation angle $\theta_{o}$ is sampled over $[0, \pi / 2]$ according to the isotropic probability density function $p_{\Theta_{0}}\left(\theta_{0}\right)=\sin \theta_{0}$.

- Step 2: A radius $r_{e q}$ for the volume equivalent sphere is sampled over ]0, $+\infty$ [ according to the log-normal distribution $p_{R_{e q}}\left(r_{e q}\right)$ given in Eq. (39) and $a=$ $(2 / 3 R)^{1 / 3} r_{e q}$ is computed.

- Step 3: $\eta_{1}$ is uniformly sampled over $[-1,1]$ and $\xi_{1}$ is uniformly sampled over $\left[-\beta\left(\theta_{0}\right)-\sqrt{1-\eta_{1}^{2}}, \beta\left(\theta_{0}\right)+\right.$ $\left.\sqrt{1-\eta_{1}^{2}}\right]$, defining a first location $\left(x_{1}, y_{1}\right)$ on the projected surface.

- Step 4: The first crossing length $l_{1}\left(\theta_{o}, r_{e q}, \eta_{1}, \xi_{1}\right)$ is computed according to Eqs. (E.2) and (E.3).

- Step 5: $\eta_{2}$ is uniformly sampled over $[-1,1]$ and $\xi_{2}$ is uniformly sampled over $\left[-\beta\left(\theta_{0}\right)-\sqrt{1-\eta_{2}^{2}}, \beta\left(\theta_{0}\right)+\right.$ $\left.\sqrt{1-\eta_{2}^{2}}\right]$, defining a second location $\left(x_{2}, y_{2}\right)$ on the projected surface.

- Step 6: The second crossing length $l_{2}\left(\theta_{0}, r_{e q}, \eta_{2}, \xi_{2}\right)$ is computed according to Eqs. (E.2) and (E.3).

- Step 7: The Monte Carlo weights $w\left(\theta_{s, 1}\right), w\left(\theta_{s, 2}\right), w\left(\theta_{s, 3}\right)$, etc. for different scattering angles $\theta_{s, 1}, \theta_{s, 2}, \theta_{s, 3}$, etc. are computed according to Eq. (E.7).

\section{Appendix F. Simplified model for large-angle scattering}

The purpose of the present Appendix (which is based on [30] but we present it here again for completeness) is to derive our simplified model for the differential scattering cross-section at large angles. Hereafter we focus on the expression of the differential scattering cross-section equation (14) averaged over size and orientation distribution

$$
\begin{aligned}
& W_{s}\left(\theta_{s}\right)=\left(\frac{k_{e}^{2}}{2 \pi}\right)^{2}\left|m_{r}-1\right|^{2} \frac{1+\cos ^{2}\left(\theta_{s}\right)}{2} \\
& \int_{4 \pi} d \vec{e}_{o} p_{\vec{E}_{o}}\left(\vec{e}_{o}\right) \int_{0}^{\infty} d r_{e q} p_{R_{e q}}\left(r_{e q}\right)\left|\int_{\mathcal{V}_{p}} d \vec{r} t(\vec{r}) h(\vec{r})\right|^{2}
\end{aligned}
$$

where we introduced the functions

$t(\vec{r})=\exp \left\{-k_{e} \kappa_{r} l^{\text {tot }}(\vec{r})\right\}$

and

$h(\vec{r})=\exp \left\{i\left[\vec{q} \cdot \vec{r}-k_{e}\left(n_{r}-1\right) l^{t o t}(\vec{r})\right]\right\}$

with $l^{\text {tot }}(\vec{r})=l^{\text {in }}\left(\vec{r}, \vec{e}_{i}, \vec{e}_{o}, r_{e q}\right)+l^{\text {out }}\left(\vec{r}, \vec{e}_{s}, \vec{e}_{o}, r_{e q}\right)$ (see Fig. 1b). In order to derive our simplified behavior of $W_{s}$ at large angles, we first write the square modulus $\left|\int_{\mathcal{V}_{p}} d \vec{r} t(\vec{r}) h(\vec{r})\right|^{2}$ in Eq. (F.1) as an integral over two locations $\vec{r}_{1}$ and $\vec{r}_{2}$ of the particule's volume $\mathcal{V}_{p}$ (in the very same manner as when deriving the Monte Carlo integral formulation of the differential scattering cross-section at small angles in Section 3.2 and Eq. (31)):

$$
\begin{aligned}
\mid \int_{\mathcal{V}_{p}} d \vec{r} & \left.\left.t(\vec{r}) h(\vec{r})\right|^{2}=\Re^{2} \int_{\mathcal{V}_{p}} d \vec{r} t(\vec{r}) h(\vec{r})\right) \\
& \left.+\mathfrak{J}^{2} \int_{\mathcal{V}_{p}} d \vec{r} t(\vec{r}) h(\vec{r})\right) \\
= & \int_{\mathcal{V}_{p}} d \vec{r}_{1} \int_{\mathcal{V}_{p}} d \vec{r}_{2} t\left(\vec{r}_{1}\right) t\left(\vec{r}_{2}\right)\left[\Re h\left(\vec{r}_{1}\right) \mathfrak{R} h\left(\vec{r}_{2}\right)\right. \\
& \left.+\mathfrak{I} h\left(\vec{r}_{1}\right) \mathfrak{I} h\left(\vec{r}_{2}\right)\right] \\
= & \int_{\mathcal{V}_{p}} d \vec{r}_{1} \int_{\mathcal{V}_{p}} d \vec{r}_{2} t\left(\vec{r}_{1}\right) t\left(\vec{r}_{2}\right) \cos \left[h\left(\vec{r}_{1}\right)-h\left(\vec{r}_{2}\right)\right]
\end{aligned}
$$

with

$t\left(\vec{r}_{1}\right) t\left(\vec{r}_{2}\right)=\exp \left[-k_{e} \kappa_{r} \Sigma l^{t o t}\right]$

and

$$
\begin{aligned}
\cos & {\left[h\left(\vec{r}_{1}\right)-h\left(\vec{r}_{2}\right)\right] } \\
& =\cos \left[k_{e}\left(2 \sin \frac{\theta_{s}}{2} \vec{q}_{u} \cdot \vec{\rho}-\left(n_{r}-1\right) \Delta l^{t o t}\right)\right]
\end{aligned}
$$

where $\vec{q}_{u}=\vec{q} /\|\vec{q}\|$ is the unit vector associated with $\vec{q}=k_{e}\left(\vec{e}_{s}-\vec{e}_{i}\right), \quad \vec{\rho}=\vec{r}_{1}-\vec{r}_{2}, \quad \Sigma l^{\text {tot }}=l^{\text {tot }}\left(\vec{r}_{1}\right)+l^{\text {tot }}\left(\vec{r}_{2}\right)$ and $\Delta l^{\text {tot }}=l^{\text {tot }}\left(\vec{r}_{1}\right)-l^{\text {tot }}\left(\vec{r}_{2}\right)$. Then, qualitatively applying the principle of the stationary phase approximation (along the line of Schiff's work [20]) we notice that the main contribution to Eq. (F.2) comes from the integration domain such that $\|\vec{\rho}\|<\left(1 / k_{e} 2 \sin \left(\theta_{s} / 2\right)\right)$. Indeed, outside of this region the integrand rapidly oscillates around zero as $\vec{\rho}$ is changed because:

- $2 \sin \left(\theta_{s} / 2\right) \vec{q}_{u} \cdot \vec{\rho}$ dominates the argument of the cosine in Eq. (F.4),

- when $\vec{r}_{1}$ and $\vec{r}_{2}$ are distant enough (i.e. when $\|\vec{\rho}\|$ is large) then $k_{e} \Delta l^{\text {tot }}$ varies in the range of $\left[0, k_{e} a\right]=[0, x]$ (where $a$ is the characteristic length of the particle and $x$ is its size parameter; $x \gg 1$ in our framework) while at the opposite, when $\vec{r}_{1} \simeq \vec{r}_{2}, k_{e} \Delta l^{\text {tot }} \simeq 0$. 
If we neglect the effect of the boundary of $\mathcal{V}_{p}$, this stationary phase region corresponding to $\vec{r}_{1}=\vec{r}_{2}$ has a volume $4 \pi /\left(3\left(k_{e} 2 \sin \theta_{s} / 2\right)^{3}\right)$ regardless of the location $\vec{r}_{1}$, and since $\vec{\rho}=\overrightarrow{0}$, we have $\Sigma l^{\text {tot }}=2 l^{\text {tot }}\left(\vec{r}_{1}\right)$ and $\Delta l^{\text {tot }}=0$. Thus, we obtain the simplification:

$$
\begin{aligned}
W_{s}\left(\theta_{s}\right) & \propto \frac{1}{\left(\sin \theta_{s} / 2\right)^{3}} \frac{1+\cos ^{2} \theta_{s}}{2} \\
& \times \int_{4 \pi} d \vec{e}_{o} p_{\vec{E}_{o}}\left(\vec{e}_{o}\right) \int_{0}^{\infty} d r_{e q} p_{R_{e q}}\left(r_{e q}\right) \\
& \times \int_{\mathcal{V}_{p}\left(\vec{e}_{o}, r_{e q}\right)} d \vec{r}_{1} \exp \left[-k_{e} \kappa_{r} 2 l^{t o t}\left(\vec{r}_{1} ; \vec{e}_{o}, r_{e q}\right)\right]
\end{aligned}
$$

Moreover, for the situations that we studied, we always recorded variations of the term

$\int_{4 \pi} d \vec{e}_{o} p_{\vec{E}_{o}}\left(\vec{e}_{o}\right) \int_{0}^{\infty} d r_{e q} p_{R_{e q}}\left(r_{e q}\right) \int_{\mathcal{V}_{p}\left(\vec{e}_{o}, r_{e q}\right)} d \vec{r}_{1}$

$\exp \left[-k_{e} \kappa_{r} 2 l^{\text {tot }}\left(\vec{r}_{1} ; \vec{e}_{o}, r_{e q}\right)\right]$

as function of $\theta_{s}$ that are negligible compared to the variations of the first term in Eq. (F.5). Therefore, we retain the following simplified behavior for the variation of $W_{s}$ as a function of $\theta_{s}$ at large angles $\left(\theta_{s}>\theta_{l}\right)$, regardless of the scatterer geometry, as a first approximation of Eq. (F.1):

$W_{s}\left(\theta_{s}\right) \propto \frac{1}{\left(\sin \theta_{s} / 2\right)^{3}} \frac{1+\cos ^{2} \theta_{s}}{2}$

We notice that this mathematical expression is also encountered in the expression of $W_{s}$ for spheroids and cylinders with the Rayleigh-Gans approximation [14,47]. On this basis, we propose to furthermore modify the backscattering model (Eq. (F.6)) as

$W_{s}\left(\theta_{s}\right)=\frac{A}{\left(\sin \theta_{s} / 2\right)^{B}} \frac{1+\cos ^{2} \theta_{s}}{2}$

in order to establish a model with two free parameters $A$ and $B$ that allow us to fulfil the continuity of $W_{s}$ at $\theta_{l}$, the normalization $\sigma_{s}=2 \pi \int_{0}^{\pi} d \theta_{s} \sin \left(\theta_{s}\right) W_{s}\left(\theta_{s}\right)$ and $W_{s}>0$, without degrading the results of the Schiff approximation for small angles (see Appendix G). Indeed, it is important here to not deteriorate the description of the small angles since they are essential for the study of photobioreactors and since they correspond to a better level of approximation. We note that all the situations treated in Section 4

\section{Appendix G. Determining the value of the parameters $A$ and $B$ in the simplified model for scattering at large angle}

In this Appendix, we give the procedure used in Figs. 5 and 6 (as well as in [30]) to determine the value or the free parameters $A$ and $B$ in Eq. (18). First the scattering cross-section $\sigma_{s}$, the differential scattering cross-section $W_{s}\left(\theta_{l}\right)$ and its cumulatice $C W_{s}\left(\theta_{l}\right)$ are evaluated at angle $\theta_{s}=\theta_{l}$ thanks to the algorithms given in Appendices D and $\mathrm{E}\left(\theta_{l}\right.$ is defined in Eq. (7)). We note $\sigma_{s}^{M C}, W_{s}^{M C}\left(\theta_{l}\right)$ and $C W_{s}^{M C}\left(\theta_{l}\right)$ these values obtained with Monte Carlo. Based on these results, the parameters $A$ and $B$ have to be adjusted to ensure that

- 1. $W_{s}$ is continuous at $\theta_{s}=\theta_{l}$ :

$$
\frac{A}{\sin ^{B}\left(\theta_{l} / 2\right)} \frac{1+\cos ^{2}\left(\theta_{l}\right)}{2}=W_{s}^{M C}\left(\theta_{l}\right)
$$

- 2. The integral of $W_{s}$ over the total solid angle is equal to $\sigma_{s}:$

$$
\begin{aligned}
\sigma_{s}^{M C}= & 2 \pi \int_{0}^{\pi} d \theta_{s} \sin \left(\theta_{s}\right) W_{s}\left(\theta_{s}\right) \\
\sigma_{s}^{M C}= & C W_{s}^{M C}\left(\theta_{l}\right) \\
& +2 \pi \int_{\theta_{l}}^{\pi} d \theta_{s} \sin \left(\theta_{s}\right) \frac{A}{\sin ^{B}\left(\theta_{s} / 2\right)} \frac{1+\cos ^{2}\left(\theta_{s}\right)}{2}
\end{aligned}
$$

where

$$
\begin{aligned}
& 2 \pi \int_{\theta_{l}}^{\pi} d \theta_{S} \sin \left(\theta_{S}\right) \frac{A}{\sin ^{B}\left(\theta_{S} / 2\right)} \frac{1+\cos ^{2}\left(\theta_{S}\right)}{2} \\
& =2 \pi A\left[-\sin ^{2-B}(\theta / 2) \frac{\left(B^{2}-6 B+8\right) \cos (2 \theta)+3 B^{2}-8(B-2) \cos (\theta)-26 B+72}{(B-2)(B-4)(B-6)}\right]_{\theta_{l}}^{\pi}
\end{aligned}
$$

From Eq. (G.1), we obtain

$$
A=2 W_{s}^{M C}\left(\theta_{l}\right) \frac{\sin ^{B}\left(\theta_{l} / 2\right)}{1+\cos ^{2}\left(\theta_{l}\right)}
$$

that is injected into Eq. (G.4). Therefore, Eq. (G.3) becomes:

$$
\sigma_{s}^{\mathrm{MC}}=C W_{s}^{\mathrm{MC}}\left(\theta_{l}\right)+\frac{4 \pi W_{s}^{\mathrm{MC}}\left(\theta_{l}\right)}{1+\cos ^{2}\left(\theta_{l}\right)} \frac{\sin ^{2}\left(\theta_{l} / 2\right)\left(\left(B^{2}-6 B+8\right) \cos \left(2 \theta_{l}\right)+3 B^{2}-8(B-2) \cos \left(\theta_{l}\right)-26 B+72\right)-\sin ^{B}\left(\theta_{l} / 2\right)\left(4 B^{2}-24 B+40\right)}{(B-2)(B-4)(B-6)}
$$

and in [30] correspond to values of $B$ that are close to 3 (more precisely $2<B<4$ ), as in Eq. (F.6) before freeing the parameters.
A Newton method is implemented in order to retrieve the value of $B$ ensuring that Eq. (G.6) is verified and finally, the value of $A$ is obtained by injecting the value retrieved for $B$ into Eq. (G.5). 


\section{References}

[1] Wriedt T. Light scattering theories and computer codes. J Quant Spectrosc Radiat Transf 2009;110(11):833-43.

[2] Kahnert F. Numerical methods in electromagnetic scattering theory. J Quant Spectrosc Radiat Transf 2003(79-80):775-824.

[3] Mishchenko MI, Travis LD, Lacis AA. Scattering, absorption, and emission of light by small particles. Cambridge: Cambridge University Press; 2002, URL 〈http://www.giss.nasa.gov/staff/mmish chenko/t_matrix.html>.

[4] Yurkin MA, Hoekstra AG. The discrete dipole approximation: an overview and recent developments. J Quant Spectrosc Radiat Transf 2007; 106(1):558-89.

[5] Yurkin MA, Hoekstra AG. The discrete-dipole-approximation code ADDA: capabilities and known limitations. J Quant Spectrosc Radiat Transf 2011;112(13):2234-47.

[6] Johnson BR. Invariant imbedding T-Matrix approach to electromagnetic scattering. Appl Opt 1988;27(23):4861-73.

[7] Moskalensky AE, Yurkin MA, Konokhova AI, Strokotov DI, Nekrasov VM, Chernyshev AV, et al. Accurate measurement of volume and shape of resting and activated blood platelets from light scattering. J Biomed Opt 2013;18(1):017001-2.

[8] Bi L, Yang P. Modeling of light scattering by biconcave and deformed red blood cells with the invariant imbedding t-matrix method. J Biomed Opt 2013;18(5):055001-2.

[9] Konokhova AI, Gelash AA, Yurkin MA, Chernyshev AV, Maltsev VP. High-precision characterization of individual E. coli cell morphology by scanning flow cytometry. Cytom Part A 2013;83(6):568-75.

[10] Bi L, Yang P. Impact of calcification state on the inherent optica properties of emiliania huxleyi coccoliths and coccolithophores. J Quant Spectrosc Radiat Transf 2015;155:10-21.

[11] Yang P, Wei H, Huang H-L, Baum BA, Hu YX, Kattawar GW, et al. Scattering and absorption property database for nonspherical ice particles in the near-through far-infrared spectral region. Appl Opt 2005;44(26):5512-23.

[12] Sharma SK, Sommerford DJ. Light scattering by optically soft particles: theory and applications. Springer Science \& Business Media; 2006.

[13] Bi L, Yang P, Kattawar GW, Hu Y, Baum BA. Scattering and absorption of light by ice particles: solution by a new physical-geometric optics hybrid method. J Quant Spectrosc Radiat Transf 2011;112(9): 1492-508.

[14] Van de Hulst HC. Light scattering by small particles. New York: Courier Corporation; 1957.

[15] Ackerman SA, Stephens GL. The absorption of solar radiation by cloud droplets: an application of anomalous diffraction theory. J Atmos Sci 1987;44(12):1574-88.

[16] He Z, Qi H, Yao Y, Ruan L. An effective inversion algorithm for retrieving bimodal aerosol particle size distribution from spectral extinction data. J Quant Spectrosc Radiat Transf 2014;149:117-27.

[17] Sharma SK. A modified Rayleigh-Gans-Debye formula for smal angle X-ray scattering by interstellar dust grains. Astrophys Space Sci 2015;357(1):80.

[18] Hammer M, Schweitzer D, Michel B, Thamm E, Kolb A. Single scattering by red blood cells. Appl Opt 1998:37(31):7410.

[19] Quirantes A, Bernard S. Light scattering by marine algae: two-layer spherical and nonspherical models. J Quant Spectrosc Radiat Transf 2004:89(1-4):311-21.

[20] Schiff L. Approximation method for short wavelength or highenergy scattering. Phys Rev 1956;104(5):1481-5.

[21] Flatau P. Scattering by irregular particles in anomalous diffraction and discrete dipole approximation [Ph.D. thesis]. Colorado State Univ.; 1992.

[22] Delatorre J, Baud G, Bézian J, Blanco S, Caliot C, Cornet J-F, et al. Monte Carlo advances and concentrated solar applications. Sol Energy 2014;103:653-81. http://dx.doi.org/10.1016/j.solener.2013.02.035.

[23] Dauchet J, Blanco S, Cornet J-F, El Hafi M, Eymet V, Fournier R. The practice of recent radiative transfer Monte Carlo advances and its contribution to the field of microorganisms cultivation in photobioreactors. J Quant Spectrosc Radiat Transf 2013;128:52-9.
24] Farges O, Bézian J, Bru H, El Hafi M, Fournier R, Spiesser C. Life-time integration using Monte Carlo Methods when optimizing the design of concentrated solar power plants. Sol Energy 2015;113:57-62.

[25] de Lataillade A, Blanco S, Clergent Y, Dufresne J, El Hafi M, Fournier R. Monte Carlo method and sensitivity estimations. J Quant Spectrosc Radiat Transf 2002;75(5):529-38.

[26] Roger M, Blanco S, El Hafi M, Fournier R. Monte Carlo estimates of domain-deformation sensitivities. Phys Rev Lett 2005;95(18): 180601.

[27] Dunn WL, Shultis JK. Exploring Monte Carlo methods. Fort Collins (USA): Elsevier; 2011

[28] Hammersley J, Handscomb DC. Monte Carlo methods. London: Chapman and Hall;1964.

[29] James F. Monte Carlo theory and practice. Rep Prog Phys 1980;43(9): 1145-89.

[30] Dauchet J, Blanco S, Cornet J-F, Fournier R. Calculation of the radiative properties of photosynthetic microorganisms. J Quant Spectrosc Radiat Transf 2015;161:60-84.

[31] Troyer M, Wiese U-J. Computational complexity and fundamental limitations to fermionic quantum Monte Carlo simulations. Phys Rev Lett 2005;94(17):170201

[32] StarWest, Edstar development environment project - codes. URL 〈http://www.edstar.Imd.jussieu.fr/codes〉.

[33] Aiba S. Growth kinetics of photosynthetic microorganisms. Adv Biochem Eng 1982;23:85-156.

[34] Pilon L, Berberoğlu H, Kandilian R. Radiation transfer in photobiological carbon dioxide fixation and fuel production by microalgae. J Quant Spectrosc Radiat Transf 2011;112(17):2639-60.

[35] Cornet J-F, Dussap C-G. A simple and reliable formula for assessment of maximum volumetric productivities in photobioreactors. Biotechnol Prog 2009;25(2):424-35.

[36] Pruvost J, Cornet JF. Knowledge models for the engineering and optimization of photobioreactors. In: Posten WC, De Gruyter C, editors, Microalgal biotechnology: potential and production; 2012. p. 181-224.

[37] Galtier M, Blanco S, Caliot C, Coustet C, Dauchet J, El Hafi M, et al. Integral formulation of null-collision Monte Carlo algorithms. J Quant Spectrosc Radiat Transf 2013;125:57-68.

[38] Assaraf R, Caffarel M. Zero-variance principle for Monte Carlo algorithms. Phys Rev Lett 1999;83(23):4682-5.

[39] Bohren CF, Huffman DR. Absorption and scattering of light by small particles. Bohren: Wiley Online Library; 1983.

[40] Xu M, Katz A. Statistical interpretation of light anomalous diffraction by small particles and its applications in bio-agent detection and monitoring. In: Kokhanovsky A, editors. Light scattering reviews, vol. 3. Berlin, Heidelberg: Springer Praxis Books (Springer); 2008. p. 27-67.

[41] Eymet V, Piaud B, Coustet C. Kspectrum: an open-source code for high-resolution molecular absorption spectra production. In: Proceedings of computational thermal radiation in participating media V conference, Albi, April 1-3, 2015; 2015.

[42] StarWest, Edstar development environment project. URL 〈http:// www.edstar.lmd.jussieu.fr $/\rangle$.

[43] Mesostar. URL 〈http://www.meso-star.com/en_index.html〉.

[44] Vaillon R, Geffrin J-M, Eyraud C, Merchiers O, Sabouroux P, Lacroix B. A new implementation of a microwave analog to light scattering measurement device. J Quant Spectrosc Radiat Transf 2011;112(11): 1753-60.

[45] Dauchet J. Analyse radiative des photobioréacteurs [Ph.D. thesis], no. 2304. Université Blaise Pascal, Clermont Ferrand II, France; 2012.

[46] Yang P, Zhang Z, Baum Ba, Huang H-L, Hu Y. A new look at anomalous diffraction theory (ADT): algorithm in cumulative projectedarea distribution domain and modified ADT. J Quant Spectrosc Radiat Transf 2004;89(1-4):421-42.

[47] Wyatt PJ. Differential light scattering: a physical method for identifying living bacterial cells. Appl Opt 1968;7(10):1879-96. 